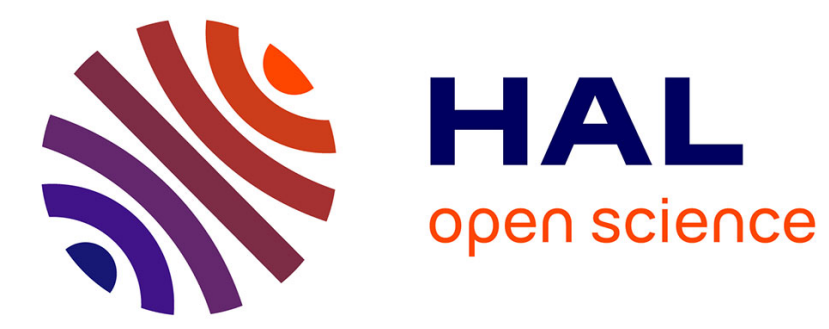

\title{
Continuous Previsions
}

Jean Goubault-Larrecq

\section{To cite this version:}

Jean Goubault-Larrecq. Continuous Previsions. Computer Science Logic, 4646, Springer Berlin Heidelberg, pp.542-557, 2007, Lecture Notes in Computer Science, 10.1007/978-3-540-74915-8_40 . hal03197352

\section{HAL Id: hal-03197352 \\ https://hal.science/hal-03197352}

Submitted on 9 Jun 2021

HAL is a multi-disciplinary open access archive for the deposit and dissemination of scientific research documents, whether they are published or not. The documents may come from teaching and research institutions in France or abroad, or from public or private research centers.
L'archive ouverte pluridisciplinaire HAL, est destinée au dépôt et à la diffusion de documents scientifiques de niveau recherche, publiés ou non, émanant des établissements d'enseignement et de recherche français ou étrangers, des laboratoires publics ou privés. 


\title{
Continuous Previsions ${ }^{\star}$
}

\author{
Jean Goubault-Larrecq \\ LSV, ENS Cachan, CNRS, INRIA Futurs \\ 61, avenue du président-Wilson, 94235 Cachan, France \\ goubaultelsv.ens-cachan.fr
}

\begin{abstract}
We define strong monads of continuous (lower, upper) previsions, and of forks, modeling both probabilistic and non-deterministic choice. This is an elegant alternative to recent proposals by Mislove, Tix, Keimel, and Plotkin. We show that our monads are sound and complete, in the sense that they model exactly the interaction between probabilistic and (demonic, angelic, chaotic) choice.
\end{abstract}

\section{Introduction}

Moggi's computational $\lambda$-calculus [17] has proved useful to define various notions of computations on top of the lambda-calculus: side-effects, input-output, continuations, non-determinism [26], probabilistic computation [20] in particular. But mixing monads is hard, and finding the "right" monad that would combine both non-determinism and probabilistic choice has taken quite some effort. (We review recent progress below.)

The purpose of this paper is to introduce simple monads that do the job well. These are monads of continuous previsions, which can be seen as continuation-style monads. The idea of considering previsions comes from economics and statistics $[5,12]$.

Outline. After stating some required preliminaries in Section 2, we recall the notion of game introduced in [6], arguing why these are natural extensions of notions of continuous valuations ( $\sim$ measures) that also accommodate demonic and angelic non-deterministic choice. These notions induce functors on Top, Cpo, Pcpo, but fail to yield monads. We analyze this failure in Section 4 by moving, through a Riesz-like representation theorem, to the new notions of collinear previsions, and previsions. We then show that indeed previsions yield strong monads, suitable to give semantics to a rich $\lambda$-calculus [17] with both probabilistic and non-deterministic choice. Finally, we show in Section 5 that our monad model is not only sound but complete.

This work is a summary of most of Chapters 10-12 of [7]. We however give the proofs of the main results in the Appendix.

Related Work. Finding a monad combining both probabilistic and non-deterministic choice can be done by using general monad combination principles. The right way to combine monads in general is open to discussion. Lüth [11] proposes to combine monads by taking their coproduct in the category of monads. This coproduct exists under relatively mild assumptions [10]. However, in general the coproduct of two monads is an inscrutable object. A simpler, explicit description can be found in specific cases. For

\footnotetext{
* Partially supported by the INRIA ARC ProNoBis. Part of Section 4 was done while the author was invited at U. Laval, Québec City, Québec, July 2004. We acknowledge their support.
} 
example, when taking coproducts of two ideal monads [3]. In particular, combining non-blocking non-determinism and probabilistic choice falls into this case. The resulting monad is relatively unenlightening, though: it is the monad of all sequences of choices, both probabilistic and non-deterministic [3, exemple 4.3].

Varacca [25] also proposed a monad combining non-determinism with probabilistic choice. Ghani and Uustalu [3] note that the above coproduct monad is close to Varacca's synchronization trees. The works closer to ours in computer science are those of Mislove [16] and Tix $[23,24]$. While this won't be entirely obvious from our definitions, we will establish a formal connection between their models and ours (Section 5). Outside computer science, previsions have their roots in economics and statistics [27]. However, we consider previsions on topological spaces, not just on sets.

This paper can be seen also be seen as a followup to [6], inasmuch as previsions are strongly tied to notions of convex and concave games.

\section{Preliminaries}

We assume the reader to be familiar with (point-set) topology, in particular topology of $T_{0}$ but not necessarily Hausdorff spaces, as encountered in domain theory. See $[4,1,15]$ for background. Let $\operatorname{int}(A)$ denote the interior of $A, \operatorname{cl}(A)$ its closure.

The Scott topology on a poset $X$, with ordering $\leq$, has as opens the upward-closed subsets $U$ (i.e., $x \in U$ and $x \leq y$ imply $y \in U$ ) such that for every directed family $\left(x_{i}\right)_{i \in I}$ having a least upper bound $\sup _{i \in I} x_{i}$ inside $U$, some $x_{i}$ is already in $U$. The way-below relation $\ll$ is defined by $x \ll y$ iff for any directed family $\left(z_{i}\right)_{i \in I}$ with a least upper bound $z$ such that $y \leq z$, then $x \leq z_{i}$ for some $i \in I$. A poset is continuous iff $\downarrow y=\{x \in X \mid x \ll y\}$ is directed, and has $x$ as least upper bound. Then every open $U$ can be written $\bigcup_{x \in U} \uparrow x$, where $\uparrow x=\{y \in X \mid x \ll y\}$.

Every topological space $X$ has a specialization quasi-ordering $\leq$, defined by: $x \leq y$ iff every open that contains $x$ contains $y . X$ is $T_{0}$ iff $\leq$ is a (partial) ordering. That of the Scott topology of a quasi-ordering $\leq$ is $\leq$ itself. A subset $A \subseteq X$ is saturated if and only if $A$ is the intersection of all opens that contain it; alternatively, iff $A$ is upward-closed in $\leq$. Every open is upward-closed. Let $\uparrow A$ denote the upward-closure of $A$ under a quasi-ordering $\leq, \downarrow A$ its downward-closure. A $T_{0}$ space is sober iff every irreducible closed subset is the closure $c l\{x\}=\downarrow x$ of a (unique) point $x$. The Hofmann-Mislove Theorem implies that every sober space is well-filtered [9]: given any filtered family of saturated compacts $\left(Q_{i}\right)_{i \in I}$ in $X$, and any open $U, \bigcap_{i \in I} Q_{i} \subseteq U$ iff $Q_{i} \subseteq U$ for some $i \in I$. In particular, $\bigcap_{i \in I} Q_{i}$ is saturated compact. $X$ is locally compact iff whenever $x \in U$ ( $U$ open) there is a saturated compact $Q$ such that $x \in$ $\operatorname{int}(Q) \subseteq Q \subseteq U$. Every continuous cpo is sober and locally compact in its Scott topology. $X$ is coherent iff the intersection of any two compacts is compact. A coherent, well-filtered locally compact space is called stably locally compact. Stably compact spaces are those that are additionally compact, and have a wonderful theory (see, e.g., [9]). We shall consider the space $\mathbb{R}$ of all reals with the Scott topology of its natural ordering $\leq$. Its opens are $\emptyset, \mathbb{R}$, and the intervals $(t,+\infty), t \in \mathbb{R}$. $\mathbb{R}$ is a stably locally compact, continuous cpo. Because we equip $\mathbb{R}$ with the Scott topology, our continuous 
functions $f: X \rightarrow \mathbb{R}$ are those which are usually called lower semi-continuous in the mathematical literature.

We call capacity on $X$ any function $\nu$ from $\mathcal{O}(X)$, the set of all opens of $X$, to $\mathbb{R}^{+}$, such that $\nu(\emptyset)=0$ (a.k.a., a set function). A game $\nu$ is a monotonic capacity, i.e., $U \subseteq V$ implies $\nu(U) \leq \nu(V)^{1}$. A valuation is a modular game $\nu$, i.e., one such that $\nu(\bar{U} \cup V)+\nu(U \cap V)=\nu(U) \cap \nu(V)$ for every opens $U, V$. A game is continuous iff $\nu\left(\bigcup_{i \in I} U_{i}\right)=\sup _{i \in I} \nu\left(U_{i}\right)$ for every directed family $\left(U_{i}\right)_{i \in I}$ of opens, and normalized iff $\nu(X)=1$. Continuous valuations have a nice theory that fits topology well [8,9].

The Dirac valuation $\delta_{x}$ at $x \in X$ is the continuous valuation mapping each open $U$ to 1 if $x \in U$, to 0 otherwise. Continuous valuations are canonically ordered by $\nu \leq \nu^{\prime}$ iff $\nu(U) \leq \nu^{\prime}(U)$ for every open $U$ of $X$.

A monad on a category $\mathbb{C}$ may be presented in several different ways. One is based on triples $(\boldsymbol{T}, \boldsymbol{\eta}, \boldsymbol{\mu})$ of an endofunctor on $\mathbb{C}$, a unit, and a multiplication natural transformation. A presentation that is easier to grasp is in terms of Kleisli triples [14]. A Kleisli triple is a triple $\left(\boldsymbol{T}, \boldsymbol{\eta},{ }_{-}^{\dagger}\right)$, where $T$ maps objects $X$ of $\mathbb{C}$ to objects $\boldsymbol{T} X$ of $\mathbb{C}$, $\eta_{X}$ is a morphism from $X$ to $T X$ for each $X$, and $f^{\dagger}$ (the extension of $f$ ) is a morphism from $\boldsymbol{T} X$ to $\boldsymbol{T} Y$ for each morphism $f: X \rightarrow \boldsymbol{T} Y$, satisfying: (1) $\boldsymbol{\eta}_{X}^{\dagger}=\mathrm{id}_{\boldsymbol{T} X}$; (2) for every $f: X \rightarrow \boldsymbol{T} Y, f^{\dagger} \circ \boldsymbol{\eta}_{X}=f$; (3) for every $g: X \rightarrow \boldsymbol{T} Y, f: Y \rightarrow \boldsymbol{T} Z$, then $f^{\dagger} \circ g^{\dagger}=\left(f^{\dagger} \circ g\right)^{\dagger}$. Kleisli triples and monads are equivalent.

\section{Continuous Games, Convexity, Concavity}

We follow [6]. A game $\nu$ on $X$ on $X$ is convex iff $\nu(U \cup V)+\nu(U \cap V) \geq \nu(U)+\nu(V)$ for every opens $U, V$. It is concave if the opposite inequality holds. Convex games are a cornerstone of economic theory $[5,18]$.

One fundamental example of a game that is not a valuation is the unanimity game $\mathfrak{u}_{A}(A \neq \emptyset)$, defined by $\mathfrak{u}_{A}(U)=1$ if $A \subseteq U, \mathfrak{u}_{A}(U)=0$ otherwise. As we argue in [6], $\mathfrak{u}_{A}$ is a natural "probability-like" description of demonic non-deterministic choice, in the sense that drawing "at random" according to $\mathfrak{u}_{A}$ means that some malicious adversary $C$ will choose an element of $A$ for you. This is perhaps best conveyed by a thought experiment. You, the honest player $\mathrm{P}$, would like to draw some element $x$ from $X$ with distribution $\nu$ (a game). Imagine you would like to know your chances of getting one from some (open) subset $U$ of $X$. If $\nu$ is a probability distribution, then your chances will be equal to $\nu(U)$. This is standard. For general $\nu$, continue to define your chances as $\nu(U)$. If $\nu=\mathfrak{u}_{A}$, and $U$ does not contain $A$, then $\nu(U)=0$, and your chances are zero: intuitively, $\mathrm{C}$ will pick an element in $A$, but outside $U$-on purpose. The only case where $\mathrm{C}$ is forced to pick an element in $A$ which will suit $\mathrm{P}$ (i.e., be in $U$, too), is when $A \subseteq U$-and then $\mathrm{P}$ will be pleased with probability one.

It is clear that $\mathfrak{u}_{A}$ is convex. It is in fact more. Call a game $\nu$ totally convex iff:

$$
\nu\left(\bigcup_{i=1}^{n} U_{i}\right) \geq \sum_{I \subseteq\{1, \ldots, n\}, I \neq \emptyset}(-1)^{|I|+1} \nu\left(\bigcap_{i \in I} U_{i}\right)
$$

\footnotetext{
${ }^{1}$ The name "game" is unfortunate, as there is no obvious relationship between this and games as they are usually handled in computer science, in particular with stochastic games. The notion stems from (cooperative) games in economics, where $X$ is the set of players, not of states.
} 
for every finite family $\left(U_{i}\right)_{i=1}^{n}$ of opens $(n \geq 1)$, where $|I|$ denotes the cardinality of $I$. A belieffunction is a totally convex game. The dual notion of total concavity is obtained by replacing $\bigcup$ by $\bigcap$ and conversely in (1), and turning $\geq$ into $\leq$. A plausibility is a totally concave game. If $\geq$ is replaced by $=$ in (1), then we retrieve the familiar inclusion-exclusion principle from statistics. In particular any (continuous) valuation is a (continuous) belief function. Clearly, any belief function is a convex game. The converses of both statements fail: $\mathrm{On} X=\{1,2,3\}$ with the discrete topology, $\mathfrak{u}_{\{1,2\}}$ is a belief function but not a valuation, and $\frac{1}{2}\left(\mathfrak{u}_{\{1,2\}}+\mathfrak{u}_{\{1,3\}}+\mathfrak{u}_{\{2,3\}}-\mathfrak{u}_{\{1,2,3\}}\right)$ is a convex game but not a belief function.

Every game of the form $\sum_{i=1}^{n} a_{i} \mathfrak{u}_{Q_{i}}$, with $a_{i} \in \mathbb{R}^{+}$, and $Q_{i}$ compact saturated and non-empty, is a continuous belief function, which we call simple belief function in [6]. When $\sum_{i=1}^{n} a_{i}=1$, drawing an element from $X$ "at random" (in the sense illustrated above) according to the simple belief function $\nu=\sum_{i=1}^{n} a_{i} \mathfrak{u}_{Q_{i}}$ intuitively corresponds to drawing one compact $Q_{i}$ at random with probability $a_{i}$, then to let the malicious adversary $\mathrm{C}$ draw some element, demonically, from $Q_{i}$ [6].

Let us turn to integration. Let $\nu$ be a game on $X$, and $f$ be continuous from $X$ to $\mathbb{R}^{+}$ (i.e., lower semi-continuous: $\mathbb{R}^{+}$comes with the Scott topology). Assume $f$ bounded, too, i.e., $\sup _{x \in X} f(x)<+\infty$. The Choquet integral of $f$ along $\nu$ is:

$$
\oint_{x \in X} f(x) d \nu=\int_{0}^{+\infty} \nu\left(f^{-1}(t,+\infty)\right) d t
$$

where the right hand side is an improper Riemann integral. This is well-defined, since $f^{-1}(t,+\infty)$ is open for every $t \in \mathbb{R}^{+}$by assumption, and $\nu$ measures opens. Also, since $f$ is bounded, the improper integrals above really are ordinary Riemann integrals over some closed intervals. The function $t \mapsto \nu\left(f^{-1}(t,+\infty)\right)$ is decreasing, and every decreasing (even non-continuous, in the usual sense) function is Riemann-integrable, therefore the definition makes sense.

Alternatively, any step function $\sum_{i=0}^{n} a_{i} \chi_{U_{i}}$, where $a_{0} \in \mathbb{R}^{+}, a_{1}, \ldots, a_{n} \in \mathbb{R}^{+}$, $X=U_{0} \supseteq U_{1} \supseteq \ldots \supseteq U_{n}$ is a decreasing sequence of opens, and $\chi_{U}$ denotes the indicator function of $U\left(\chi_{U}(x)=1\right.$ if $x \in X, \chi_{U}(x)=0$ otherwise) is continuous: its integral along $\nu$ then equals $\sum_{i=0}^{n} a_{i} \nu\left(U_{i}\right)$-for any game $\nu$. It is well-known that every bounded continuous function $f$ can be written as the least upper bound of a sequence of step functions $f_{K}=a+\frac{1}{2^{K}} \sum_{k=1}^{\left\lfloor(b-a) 2^{K}\right\rfloor} \chi_{f^{-1}\left(a+\frac{k}{2^{K}},+\infty\right)}(x), K \in \mathbb{N}$, where $a=$ $\inf _{x \in X} f(x), b=\sup _{x \in X} f(x)$. Then the integral of $f$ along $\nu$ is the least upper bound of the increasing sequence of the integrals of $f_{K}$ along $\nu$.

The main properties of Choquet integration are as follows. First, the integral is increasing in its function argument: if $f \leq g$ then the integral of $f$ along $\nu$ is less than or equal to that of $g$ along $\nu$. If $\nu$ is continuous, then integration is also Scott-continuous in its function argument. The integral is also monotonic and Scott-continuous in the game $\nu$. Integration is linear in the game, too, so integrating along $\sum_{i=1}^{n} a_{i} \nu_{i}$ is the same as taking the integrals along each $\nu_{i}$, and computing the obvious linear combination. However, Choquet integration is not linear in the function integrated, unless the game $\nu$ is a valuation. Still, it is positively homogeneous: integrating $\alpha f$ for $\alpha \in \mathbb{R}^{+}$yields $\alpha$ 
times the integral of $f$. And it is additive on comonotonic functions $f, g: X \rightarrow \mathbb{R}$ (i.e., there is no pair $x, x^{\prime} \in X$ such that $f(x)<f\left(x^{\prime}\right)$ and $\left.g(x)>g\left(x^{\prime}\right)\right)$.

Returning to the example of a simple belief function $\nu=\sum_{i=1}^{n} a_{i} \mathfrak{u}_{Q_{i}}$, the properties above imply that the integral of $f$ along $\nu$ is $\sum_{i=1}^{n} a_{i} \min _{x \in Q_{i}} f(x)$ [6, Proposition 1]. (Note that $f(x)$ indeed attains its minimum over $Q_{i}$, which is compact.) Another way to read this is as follows. Imagine $\mathrm{P}$ publishes how much money, $f(x)$, she would earn if you picked $x$. When $\sum_{i=1}^{n} a_{i}=1$, it is legitimate to say that the integral of $f$ along $\nu$ should be some form of expected income. The formula above states that, when $\nu$ is a simple belief function, your expected income is exactly what you would obtain on average by drawing $Q_{i}$ at random with probability $a_{i}$, then letting the malicious adversary C pick some element of $Q_{i}$ for you-minimizing your earnings $f(x)$. In other words, integrating along a simple belief function computes average min-payoffs.

This can be generalized to all continuous, not just simple, belief functions [6, Theorem 4]. More precisely, the space $\mathbf{C d}_{\leq 1}(X)$ of all continuous belief functions $\nu$ on $X$ such that $\nu(X) \leq 1$ is isomorphic to the space $\mathbf{V}_{\leq 1}(Q(X))$ of continuous valuations $\nu^{*}$ (of total mass at most 1 ) over the Smyth powerdomain $Q(X)$ of $X$, provided $X$ is wellfiltered and locally compact. $Q(X)$ is the cpo of non-empty compact saturated subsets of $X$, ordered by reverse inclusion $\supseteq$, and is a model of demonic non-determinism. (A similar result holds for normalized games and valuations $\nu$, i.e., such that $\nu(X)=1$ : $\nu \mapsto \nu^{*}$ is again an isomorphism from $\mathbf{C d}_{1}(X)$ to $\mathbf{V}_{1}(\mathcal{Q}(X))$.) The construction of $\nu^{*}$ from $\nu$ is relatively difficult, however it is noteworthy that when $\nu=\sum_{i=1}^{n} a_{i} \mathfrak{u}_{Q_{i}}$ is simple, then $\nu^{*}$ is exactly the simple valuation $\sum_{i=1}^{n} a_{i} \delta_{Q_{i}}$, which describes the choice of an element $Q_{i}$ at random with probability $a_{i}$, as intuition would have it.

Similarly, the space $\mathbf{P b}_{\leq 1}(X)$ of all continuous plausibilities (which are all concave) $\nu$ with $\nu(X) \leq 1$ is isomorphic to $\mathbf{V}_{\leq 1}\left(\mathcal{H}_{u}(X)\right)$ when $X$ is stably locally compact, and where $\mathcal{H}_{u}(X)$ is the topological Hoare powerdomain. The latter is used to model angelic non-determinism. The corresponding simple plausibilities are of the form $\sum_{i=1}^{n} a_{i} \mathfrak{e}_{F_{i}}$, where $F_{i}$ is a non-empty closed subset of $X$ (an element of $\mathcal{H}_{u}(X)$ ), and the example game $\mathfrak{e}_{F}$ is defined so that $\mathfrak{e}_{F}(U)=1$ if $F$ meets $U, \mathfrak{e}_{F}(U)=0$ otherwise: in this case $\mathrm{C}$ tries to help you, by finding some element in $U$ that would also be in $F$, if possible.

Recall that every belief function is convex. One may show that Choquet integration along $\nu$ is super-additive (the integral of $f+g$ is at least that of $f$ plus that of $g$ ) when $\nu$ is convex, and sub-additive (the integral of $f+g$ is at most that of $f$ plus that of $g$ ) when $\nu$ is concave. See [5] for the finite case, [7, chapitre 4] for the topological case.

In the sequel, let $\mathbf{J}(X), \nabla \mathbf{J}(X), \triangle \mathbf{J}(X)$ be the spaces of plain, convex and concave continuous games respectively ("plain" meaning with no added property).

\section{Continuous Previsions}

For any space $X$, let $\left\langle X \rightarrow \mathbb{R}^{+}\right\rangle$be the space of all bounded continuous functions from $X$ to $\mathbb{R}^{+}$, with the Scott topology. Each continuous game $\nu$ on $X$ gives rise to a functional $\alpha_{\mathcal{C}}(\nu)$ from $\left\langle X \rightarrow \mathbb{R}^{+}\right\rangle$to $\mathbb{R}^{+}$, mapping $f$ to its Choquet integral along $\nu$.

Think of $f(x)$ again as defining how much money if $x$ is chosen from $X$ by some computation process. We intentionally leave the notion of computation process unde- 
fined. This may be the process of drawing "at random" along a game $\nu$, as in Section 3. In the sequel, we shall explore the view that $x$ is the output of an arbitrary program, defined in some non-deterministic and probabilitic functional language. I.e., any program returns a value $x$ ( $\perp$ on non-termination, say), and if so $\mathrm{P}$ earns $f(x)$. For purely probabilistic programs (no non-deterministic choice), a prevision $F$ is essentially a function mapping earning functions $f$ to their average value $F(f)$, over all possible executions. Slightly more generally, for any belief function $\nu$, there is a prevision $\alpha_{\mathfrak{C}}(\nu)$ that maps each $f \in\left\langle X \rightarrow \mathbb{R}^{+}\right\rangle$to the average min-payoff we get when our final earnings are given by $f$.

Milking out the properties of $\alpha_{\mathcal{C}}(\nu)$, we arrive at:

Definition 1 (Prevision). A prevision is a functional $F$ from $\left\langle X \rightarrow \mathbb{R}^{+}\right\rangle$to $\mathbb{R}^{+}$such that $F$ is positively homogeneous (for every $\alpha \geq 0, F(\alpha f)=\alpha F(f)$ ), and monotonic (if $f \leq g$ [pointwise], then $F(f) \leq F(g)$ ).

$F$ is a lower prevision if moreover $F$ is super-additive, i.e., $F(f+g) \geq F(f)+F(g)$. $F$ is an upper prevision iff $F$ is sub-additive: $F(f+g) \leq F(f)+F(g) . F$ is collinear iff $F$ is additive on comonotonic pairs, i.e., if whenever $f$ and $g$ are comonotonic, then $F(f+g)=F(f)+F(g)$. A prevision $F$ is linear iff $F(f+g)=F(f)+F(g)$ for every $f, g \in\left\langle X \rightarrow \mathbb{R}^{+}\right\rangle$.

Finally, $F$ is continuous iff it is Scott-continuous: for every directed family $\left(f_{i}\right)_{i \in I}$ of bounded continuous functions with least upper bound $f, F\left(\sup _{i \in I} f_{i}\right)=\sup _{i \in I} F\left(f_{i}\right)$.

We write $\mathbf{P}(X), \nabla \mathbf{P}(X), \triangle \mathbf{P}(X)$ respectively the spaces of all continuous previsions, of continuous lower previsions, of continuous upper previsions equipped with the Scott topology of the pointwise ordering $\leq$. The spaces $\mathbf{P}^{*}(X), \nabla \mathbf{P}^{*}(X), \triangle \mathbf{P}^{*}(X)$ will be the subspaces of those that are collinear.

We do not quite follow standard naming conventions. Standardly [27], a lower prevision is just a real-valued functional. Coherent lower previsions (taking a more readable definition from [13]) are those $F$ such that $F(f) \geq \sum_{i=1}^{n} \lambda_{i} F\left(f_{i}\right)+\lambda_{0}$ whenever $f \geq \sum_{i=1}^{n} \lambda_{i} f_{i}+\lambda_{0}, \lambda_{i}>0, \lambda_{0} \in \mathbb{R}$. In our case, we reserve the "lower" adjective, so as to have a dual notion of upper prevision.

It is clear that any continuous game $\nu$ defines a continuous collinear prevision $\alpha_{\mathfrak{e}}(\nu)$. Moreover, if $\nu$ is convex, then $\alpha_{\mathfrak{e}}(\nu)$ is lower, and if $\nu$ is concave, then $\alpha_{\mathfrak{e}}(\nu)$ is upper. The following isomorphism result, akin to Riesz' Representation Theorem, is known as Schmeidler's Theorem for convex games on discrete topologies. Let $\gamma_{\mathfrak{e}}(F)$, for any prevision $F$, be the capacity $\nu$ such that $\nu(U)=F\left(\chi_{U}\right)$ for every open $U$ of $X$. Order previsions pointwise, then:

Theorem 1. $\alpha_{\mathcal{e}} \dashv \gamma_{\mathcal{C}}$ is a Galois injection from (plain, convex, concave) games into (plain, lower, upper) collinear previsions. That is, $\alpha_{\mathrm{e}}$ and $\gamma_{\mathrm{e}}$ are monotonic, $\alpha_{\mathrm{e}}\left(\gamma_{\mathrm{e}}(F)\right) \leq$ $F$ for every collinear prevision $F$, and $\gamma_{\mathfrak{e}}\left(\alpha_{\mathfrak{e}}(\nu)\right)=\nu$ for every game $\nu$.

Moreover, when restricted to continuous previsions and games, $\alpha_{\mathcal{e}}$ and $\gamma_{\mathfrak{e}}$ define an isomorphism between $\mathbf{J}(X)$ and $\mathbf{P}^{*}(X)$, between $\nabla \mathbf{J}(X)$ and $\nabla \mathbf{P} *(X)$, between $\triangle \mathbf{J}(X)$ and $\triangle \mathbf{P}^{*}(X)$.

Proof. That $\gamma_{\mathrm{e}}(F)$ is a game for any prevision is easy. When $F$ is lower, note that $\chi_{U \cup V}$ and $\chi_{U \cap V}$ are comonotonic, and $\chi_{U \cup V}+\chi_{U \cap V}=\chi_{U}+\chi_{V}$. So $\gamma_{e}(F)(U \cup$ 
$V)+\gamma_{\mathrm{e}}(F)(U \cap V)=F\left(\chi_{U \cup V}+\chi_{U \cap V}\right)($ since $F$ is collinear $)=F\left(\chi_{U}+\chi_{V}\right) \geq$ $F\left(\chi_{U}\right)+F\left(\chi_{V}\right)$ (since $F$ is super-additive) $=\gamma_{\mathrm{e}}(F)(U)+\gamma_{\mathrm{e}}(F)(V)$. Similarly, $\gamma_{\mathrm{e}}(F)$ is concave if $F$ is upper.

For the converse, we first show that: (A) for any collinear prevision $F$ on $X$, for any step function $f$, written $a+\sum_{i=1}^{m} a_{i} \chi_{U_{i}}$ with $U_{1} \supseteq \ldots \supseteq U_{m}, a \in \mathbb{R}, a_{1}, \ldots, a_{m} \in$ $\mathbb{R}^{+}$, then the Choquet integral of $f$ along $\gamma_{\mathfrak{C}}(F)$ equals $F(f)$. This is an easy exercise as soon as one realizes that $\sum_{i=0}^{k-1} a_{i} \chi_{U_{i}}$ and $a_{k} \chi_{U_{k}}$ are comonotonic for every $k, 1 \leq k \leq$ $m$. The equality $\gamma_{\mathfrak{e}}\left(\alpha_{\mathfrak{C}}(\nu)\right)(U)=\nu(U)$ is obvious, $\alpha_{\mathfrak{e}}$ and $\gamma_{\mathfrak{e}}$ are clearly monotonic. To show that $\alpha_{\mathfrak{C}}\left(\gamma_{\mathfrak{e}}(F)\right) \leq F$, we must show that the Choquet integral of $f$ along $\gamma_{\mathrm{e}}(F)$ is less than or equal to $F(f)$. Using the step functions $f_{K}, K \in \mathbb{N}$, by (A) the Choquet integral of $f_{K}$ is less than or equal to $F\left(f_{K}\right)$. The least upper bound of the Choquet integrals of $f_{K}, K \in \mathbb{N}$ is that of $f$, and the least upper bound of $F\left(f_{K}\right)$ is at most $F(f)$. So $\alpha_{\mathfrak{C}}\left(\gamma_{\mathfrak{e}}(F)\right)(f) \leq F(f)$. When $F$ is continuous, the least upper bound of $F\left(f_{K}\right)$ is exactly $F(f)$, whence $\alpha_{\mathcal{C}}\left(\gamma_{\mathcal{C}}(F)\right)=F$.

One easy, well-known consequence of this is that $\alpha_{\mathcal{e}}$ and $\gamma_{\mathcal{e}}$ define an order isomorphism between the space $\mathbf{V}(X)$ of continuous valuations and that $\mathbf{P}^{\triangle}(X)$ of continuous linear previsions ([8, Theorem 6.2], [22, Satz 4.16]). Intuitively, any continuous game $\nu$ gives rise to a continuous collinear prevision $\alpha_{\mathfrak{e}}(\nu)$ that computes a generalized form of expectation along $\nu$, and every continuous collinear prevision arises this way.

It is easy to check that $\mathbf{J}, \nabla \mathbf{J}, \triangle \mathbf{J}, \mathbf{V}, \mathbf{P}^{\star}, \nabla \mathbf{P}^{\star}, \triangle \mathbf{P}^{*}, \mathbf{P}^{\triangle}$ define functors $T$ from $\boldsymbol{T} o p$ to $\boldsymbol{T} o p$, where $\boldsymbol{T} \boldsymbol{T} \boldsymbol{p}$ is the category of topological spaces.

To define a monad structure on $T$, we need a unit

$\eta_{X}: X \rightarrow \boldsymbol{T} X$, natural in $X$. This is defined by

$\boldsymbol{\eta}_{X}(x)=\delta_{x}$. However, there is general no extension $f^{\dagger}(\nu)(V)=\oint_{x \in X} f(x)(V) d \nu$

$f^{\dagger}$ of $f: X \rightarrow T Y$. The natural candidate is:

when $\boldsymbol{T}$ is a game functor $(\mathbf{J}, \nabla \mathbf{J}, \triangle \mathbf{J}, \mathbf{V})$, or $f^{\dagger}(F)(h)=F(\lambda x \in X \cdot f(x)(h))$ when $\boldsymbol{T}$ is a prevision functor $\left(\mathbf{P}^{*}, \nabla \mathbf{P}^{*}, \triangle \mathbf{P}^{*}, \mathbf{P}^{\triangle}\right)$. While this indeed works when $\boldsymbol{T}=\mathbf{V}$ [8, Section 4.2], or when $\boldsymbol{T}=\mathbf{P}^{\triangle}$ using the isomorphism between $\mathbf{V}$ and the latter, it fails for the other functors. To understand why, take $T=\nabla \mathbf{P}^{*}$, and consider $X=\{1,2\}, Y=\left\{*_{11}, *_{12}, *_{21}, *_{22}\right\}$ (with their discrete topologies), $F=$ $\alpha_{\mathcal{C}} \mathfrak{u}_{\{1,2\}}$, i.e., $F(h)=\min (h(1), h(2))$ for every $h: Y \rightarrow \mathbb{R}^{+}, f: X \rightarrow \boldsymbol{T Y}$ defined by $f(1)=\alpha_{\mathcal{C}}\left(3 / 4 \delta_{*_{11}}+1 / 4 \delta_{*_{12}}\right)$ and $f(2)=\alpha_{\mathcal{C}}\left(1 / 3 \delta_{*_{21}}+2 / 3 \delta_{*_{22}}\right)$, so that $f(1)(h)=3 / 4 h\left(*_{11}\right)+1 / 4 h\left(*_{12}\right)$ and $f(2)(h)=1 / 3 h\left(*_{21}\right)+2 / 3 h\left(*_{22}\right)$ for every $h: Y \rightarrow \mathbb{R}^{+}$. Let $h$ and $h^{\prime}$ be defined by: $h\left(*_{11}\right)=0.3, h\left(*_{12}\right)=h\left(*_{22}\right)=0.1$, $h\left(*_{21}\right)=0.7, h^{\prime}\left(*_{11}\right)=0.5, h^{\prime}\left(*_{12}\right)=h^{\prime}\left(*_{22}\right)=0, h^{\prime}\left(*_{21}\right)=0.7$, then $f^{\dagger}(F)(h)=$ $0.25, f^{\dagger}(F)\left(h^{\prime}\right)=0.233 \ldots, f^{\dagger}(F)\left(h+h^{\prime}\right)=0.533 \ldots$, but $f^{\dagger}(F)(h)+f^{\dagger}(F)\left(h^{\prime}\right)=$ $0.4833 \ldots \neq f^{\dagger}(F)\left(h+h^{\prime}\right)$, although $h$ and $h^{\prime}$ are comonotonic. In other words, ${ }^{\dagger}$ does not preserve collinearity.

In everyday terms, collinear previsions, or more specifically belief functions represent a process where $P$ draws at random first, then $C$ chooses non-deterministically [6]. The example above is a non-deterministic choice (among $\{1,2\}$ ) followed by probabilistic choices. In other words, the non-deterministic player $C$ plays first, then only the probabilistic player $P$. But it is well-known that you cannot permute non-deterministic and probabilistic choices, and the example above only serves to restate this. 
Our cure is simple: drop the collinearity condition. We shall therefore consider monads of continuous (plain, lower, upper) previsions. Let Posc be the category of posets with Scott-continuous maps, $C p o$ its full subcategory of cpos. We consider posets equipped with their Scott topology, whence these two categories are full subcategories of $\mathbf{T o p}$. Note that $\mathbf{P}(X), \nabla \mathbf{P}(X), \triangle \mathbf{P}(X)$ are only posets, not cpos.

Theorem 2. Define $T X$ as $\mathbf{P}(X)$, resp. $\nabla \mathbf{P}(X)$, resp. $\triangle \mathbf{P}(X)$. Let $\boldsymbol{\eta}_{X}(x)=\lambda h \in$ $\left\langle X \rightarrow \mathbb{R}^{+}\right\rangle \cdot h(x)$, and $f^{\dagger}(F)(h)=F(\lambda x \in X \cdot f(x)(h))$ for every $f: X \rightarrow \boldsymbol{T} Y$. Then $\boldsymbol{T}$ is a monad on Top, i.e., $\left(\boldsymbol{T}, \boldsymbol{\eta},{ }_{-}^{\dagger}\right)$ is a Kleisli triple. On Posc, $\boldsymbol{T}$ is a strong monad: $\boldsymbol{t}_{X, Y}: X \times \boldsymbol{T} Y \rightarrow \boldsymbol{T}(X \times Y)$ defined as $\boldsymbol{t}_{X, Y}(x, F)(h)=F(\lambda y \in Y \cdot h(x, y))$ is a tensorial strength.

Proof. We must first show that, for every $f: X \rightarrow T Y, f^{\dagger}$ is indeed a continuous map from $\boldsymbol{T} X$ to $\boldsymbol{T} Y$. Foremost, we must make sure that for every continuous (plain, lower, upper) prevision $F$ on $X, f^{\dagger}(F)$ is a continuous (plain, lower, upper) prevision on $Y$. This is easy, but relatively tedious verification. Now note that the formulae defining $\boldsymbol{\eta}$, ${ }_{-}^{\dagger}, t$ are exactly the formulae defining the continuation monad [17]. It follows that the Kleisli triple axioms also hold in our case.

Contrarily to what might be expected, $\boldsymbol{t}_{X, Y}$ is not defined on all of $\boldsymbol{T} o p$ - it may fail to be continuous. On Posc, this is repaired by the fact that a function of two arguments is continuous iff it is continuous in each argument separately (a fact that fails in Top). The tensorial strength equations [17] are checked as for the continuation monad.

That the formulae for unit, extension, and tensorial strength are the same as for the continuation monad is no accident. Imagine $F \in \boldsymbol{T} X$ is the semantics of a (probabilistic and non-deterministic) program expected to return a result $x$ of type $X$. As we have already argued, when $F=\alpha_{\mathrm{e}}(P)$, with $P$ a continuous valuation, then $F(h)$ is the average payoff, defined as the (Choquet) integral of $h(x)$ along $P$. When $F=\alpha_{\mathbb{C}}(\nu)$ with $\nu$ a continuous belief function, then $F(h)$ is the average min-payoff, where minima are taken over (demonically) non-deterministic choices. When $F$ is not collinear, then more complicated "averaging" processes are involved. In particular, we allow taking means of mins of means of mins... representing plays where $P, C, P, C, \ldots$ take turns. The fact that arbitrarily many turns can be chained in a (not necessarily collinear) prevision will be a consequence of the fact that prevision functors define monads, and in particular have a well-defined multiplication. This is standard in the monadic approach to side-effects [17]: multiplication is the key to defining sequential composition-here, of plays.

More explicitly, take $n$ continuous functions $f_{1}: X_{0} \rightarrow \boldsymbol{T} X_{1}, f_{2}: X_{1} \rightarrow \boldsymbol{T} X_{2}$, $\ldots, f_{n}: X_{n-1} \rightarrow \boldsymbol{T} X_{n}$. Then, when $\boldsymbol{T}$ is a monad, $f_{n}^{\dagger} \circ f_{n-1}^{\dagger} \circ \ldots \circ f_{2}^{\dagger} \circ f_{1}$ : $X_{0} \rightarrow \boldsymbol{T} X_{n}$ is the sequential composition of $f_{1}, f_{2}, \ldots, f_{n-1}, f_{n}$ in this order: given $x_{0} \in X_{0}$, the process $f_{1}\left(x_{0}\right)$ computes some element $x_{1} \in X_{1}$ (in our case, by drawing it "at random", say; deterministic computations are of course allowed, too), then $f_{2}\left(x_{1}\right)$ computes some $x_{2} \in X_{2}$, etc. The monad laws then typically say that composing with the idle process $\eta_{X}: X \rightarrow T X$ does nothing, and that sequential composition is associative.

While Theorem 2 then establishes a form of soundness (which we shall make more precise below), the goal of the next sections will be to show that the prevision axioms are 
complete, in the sense that there is no junk: every continuous (lower, upper) prevision is a mix of (demonic, angelic) non-deterministic and probabilistic choices.

One may wonder what the equivalent of normalized games $(\nu(X)=1)$ and subnormalized games $(\nu(X) \leq 1)$ would be through the correspondence of Theorem 1 . Requiring $F\left(\chi_{X}\right)$ to equal (resp. less than or equal to) 1 is the obvious choice. However, this is not preserved by _ ${ }^{\dagger}$ when $F$ is not collinear. So we define:

Definition 2. A prevision $F$ on $X$ is normalized, resp. sub-normalized, iff for every $f \in\left\langle X \rightarrow \mathbb{R}^{+}\right\rangle$, for every $a \in \mathbb{R}^{+}, F(a+f)=a+F(f)($ resp. $F(a+f) \leq a+F(f))$.

We let $\mathbf{J}_{1}(X), \nabla \mathbf{P}_{1}^{\star}(X), \nabla \mathbf{P}_{1}(X), \ldots$, be the subspaces of normalized games/previsions, and $\mathbf{J}_{\leq 1}(X), \nabla \mathbf{P}_{\leq 1}^{\sharp}(X), \nabla \mathbf{P}_{\leq 1}(X), \ldots$, those of sub-normalized games/previsions.

Proposition 1. Theorem 1 again holds for normalized (continuous) games and previsions, and for sub-normalized (continuous) games and previsions.

Now the spaces of sub-normalized and normalized continuous previsions are cpos. The spaces of sub-normalized continuous previsions are pointed, i.e., they have a least element $\perp$, the constant 0 function. If $X$ is itself pointed, then the spaces of normalized continuous previsions are pointed, too, with least element $\alpha_{\mathfrak{C}}\left(\delta_{\perp}\right)$ (a continuous linear prevision). The latter maps $h \in\left\langle X \rightarrow \mathbb{R}^{+}\right\rangle$to $h(\perp)$. Let $C$ po the category of cpos, Pcpo that of pointed cpos. It follows:

Proposition 2. Let $\mathbf{T} X$ be defined as $\mathbf{P}_{\leq 1}(X), \nabla \mathbf{P}_{\leq 1}(X), \triangle \mathbf{P}_{\leq 1}(X) \mathbf{P}_{1}(X), \nabla \mathbf{P}_{1}(X)$, or $\triangle \mathbf{P}_{1}(X) .(\boldsymbol{T}, \boldsymbol{\eta}, \boldsymbol{\mu}, \boldsymbol{t})$ is a strong monad on $\mathbf{C p o}$ and on $\mathbf{P c p o}$.

Theorem 2 allows us to give a semantics to a $\lambda$-calculus with both probabilistic and non-deterministic choices. Consider the syntax of terms and types:

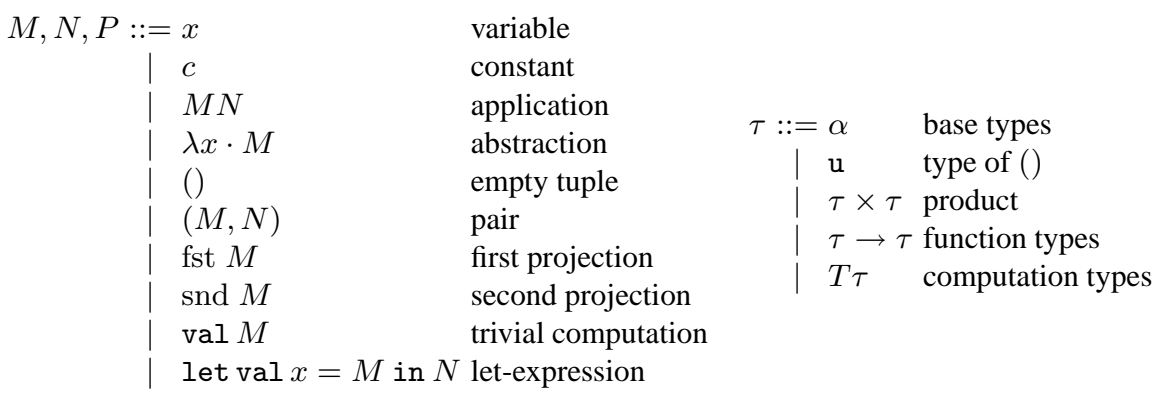

The typing rules, as well as the categorical semantics in a let-CCC, are standard [17]. Note that $\boldsymbol{C p o}$ and $\boldsymbol{P} \boldsymbol{c p o}$ are Cartesian-closed. Together with the strong monads of Proposition 2, they form let-CCCs. The typing rules for computation types are: if $\Gamma \vdash$ $M: \tau$ then $\Gamma \vdash \operatorname{val} M: T \tau$; and if $\Gamma \vdash M: T \tau_{1}$ and $\Gamma, x: \tau_{1} \vdash N: T \tau_{2}$ then $\Gamma \vdash$ let $\operatorname{val} x=M$ in $N: T \tau_{2}$.

As should be expected, the semantics has a strong continuation flavor. For each

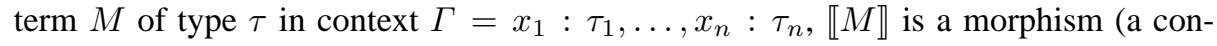
tinuous map) from $\llbracket \Gamma \rrbracket=\llbracket \tau_{1} \rrbracket \times \ldots \times \llbracket \tau_{n} \rrbracket$ to $\llbracket \tau \rrbracket$. The cases for val and let are

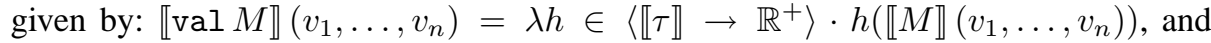
$\llbracket$ let $\operatorname{val} x=M$ in $N \rrbracket\left(v_{1}, \ldots, v_{n}\right)=\lambda h \in\left\langle\llbracket \tau_{2} \rrbracket \rightarrow \mathbb{R}^{+}\right\rangle \cdot \llbracket M \rrbracket\left(v_{1}, \ldots, v_{n}\right)(\lambda v \in$ 
$\left.\llbracket \tau_{1} \rrbracket \cdot \llbracket N \rrbracket\left(v_{1}, \ldots, v_{n}, v\right)(h)\right)$. Let bool be a base type, with $\llbracket$ bool $\rrbracket=\mathbb{S}$, where $\mathbb{S}=\{0,1\}$ is Sierpiński space $(0<1)$. Constants $c$ may include a least fixpoint operator in Pcpo, the Boolean constants false, true, a case construct case : bool $\times \tau \times \tau \rightarrow \tau$

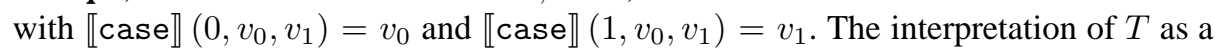
monad of previsions allows us, additionally, to give meaning to a coin-flipping op-

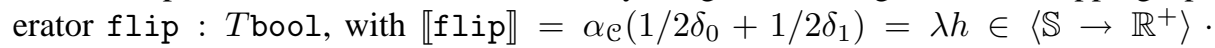
$1 / 2(h(0)+h(1))$, and a non-deterministic choice operator amb : Tbool. When $\boldsymbol{T}$ is $\nabla \mathbf{P}_{1}$, amb is the demonic choice (of a Boolean): $\llbracket a m b \rrbracket=\alpha_{\mathcal{C}}\left(\mathfrak{u}_{\{0,1\}}\right)=\lambda h \in$ $\left\langle\mathbb{S} \rightarrow \mathbb{R}^{+}\right\rangle \cdot \min (h(0), h(1))$ (the chosen Boolean $x$ is the one that minimizes payoff $h(x))$. When $\boldsymbol{T}$ is $\Delta \mathbf{P}_{1}$, we get angelic choice: $\llbracket \mathrm{amb} \rrbracket=\alpha_{\mathcal{C}}\left(\mathfrak{u}_{\{0,1\}}\right)=\lambda h \in\langle\mathbb{S} \rightarrow$ $\left.\mathbb{R}^{+}\right\rangle \cdot \max (h(0), h(1))$ (maximize payoff).

One might think that letting $\boldsymbol{T}$ be $\mathbf{P}_{1}$ would lead to chaotic choice. This certainly accommodates both demonic (min) and angelic choice (max). However, $\mathbf{P}_{1}$ is a very large space, and seems to contain objects that do not correspond to any mixture of probabilistic and non-deterministic choice. The right notion is suggested by [7, section 7.5].

Definition 3 (Fork). A fork on $X$ is any pair $F=\left(F^{-}, F^{+}\right)$where $F^{-}$is a lower prevision, $F^{+}$is an upper prevision, and for any $h, h^{\prime} \in\left\langle X \rightarrow \mathbb{R}^{+}\right\rangle$,

$$
F^{-}\left(h+h^{\prime}\right) \leq F^{-}(h)+F^{+}\left(h^{\prime}\right) \leq F^{+}\left(h+h^{\prime}\right)
$$

$F$ is continuous, resp. normalized, sub-normalized, collinear, whenever both $F^{-}$and $F^{+}$are.

While the above definition was found from purely mathematical arguments, Walley [27, Section 2] defines essentially the same notion in finance. However, we allow any pair $\left(F^{-}, F^{+}\right)$satisfying these conditions to be a fork. Walley only observes that whenever $F^{-}$is a coherent prevision (in his sense), on a discrete space, then letting $F^{+}(h)=$ $-F^{-}(-h)$ yields a fork $\left(F^{-}, F^{+}\right)$.

One may think of $F^{-}$as the pessimistic part of $F$, which will give us the least expected payoff, while $F^{+}$is the optimistic part, yielding the greatest expected payoff. Taking $h^{\prime}=0$ in (3) shows indeed that $F^{-}(h) \leq F^{+}(h)$ for each $h$. Let $\mathbf{F}(X)$ be the space of continuous forks on $X$, ordered by $\leq \times \leq$. The subspaces $\mathbf{F}_{1}(X)$ and $\mathbf{F}_{\leq 1}(X)$ of normalized and sub-normalized forks are cpos. The latter is pointed (with least element $(0,0))$ and the former is as soon as $X$ is (with least element $\left(\alpha_{\mathfrak{e}}\left(\delta_{\perp}\right), \alpha_{\mathfrak{C}}\left(\delta_{\perp}\right)\right)$ ). The semantics is essentially the pairing of two continuation semantics, e.g., \val $M \rrbracket\left(v_{1}, \ldots\right.$, $\left.v_{n}\right)=\left(F^{-}, F^{+}\right)$, where $F^{-}=F^{+}=\lambda h \in\left\langle\llbracket \tau \rrbracket \rightarrow \mathbb{R}^{+}\right\rangle \cdot h\left(\llbracket M \rrbracket\left(v_{1}, \ldots, v_{n}\right)\right)$ (a linear prevision); \let val $x=M$ in $N \rrbracket\left(v_{1}, \ldots, v_{n}\right)=\left(\lambda h \in\left\langle\llbracket \tau_{2} \rrbracket \rightarrow \mathbb{R}^{+}\right\rangle\right.$. $\left.F^{-}\left(\lambda v \in \llbracket \tau_{1} \rrbracket \cdot F_{v}^{-}(h)\right), \lambda h \in\left\langle\llbracket \tau_{2} \rrbracket \rightarrow \mathbb{R}^{+}\right\rangle \cdot F^{+}\left(\lambda v \in \llbracket \tau_{1} \rrbracket \cdot F_{v}^{+}(h)\right)\right)$, where $\left(F^{-}, F^{+}\right)=\llbracket M \rrbracket\left(v_{1}, \ldots, v_{n}\right)$ and $\left(F_{v}^{-}, F_{v}^{+}\right)=\llbracket N \rrbracket\left(v_{1}, \ldots, v_{n}, v\right)$. The constants with the most interesting semantics are amb, where $\operatorname{amb} \rrbracket=\left(\lambda h \in\left\langle\mathbb{S} \rightarrow \mathbb{R}^{+}\right\rangle\right.$. $\left.\min (h(0), h(1)), \lambda h \in\left\langle\mathbb{S} \rightarrow \mathbb{R}^{+}\right\rangle \cdot \max (h(0), h(1))\right)$ (i.e., it computes both pessimistic and optimistic outcomes), and flip, where $\llbracket$ flip $\rrbracket=\left(F^{-}, F^{+}\right)$with $F^{-}=F^{+}=$ $\lambda h \in\left\langle\mathbb{S} \rightarrow \mathbb{R}^{+}\right\rangle \cdot 1 / 2(h(0)+h(1))$. For the rest of the language, we rely on [17] and:

Proposition 3. Let $\mathbf{T} X$ be defined as $\mathbf{F}(X), \mathbf{F}_{\leq 1}(X)$, or $\mathbf{F}_{1}(X)$. Let $\boldsymbol{\eta}_{X}(x)=\left(F^{-}, F^{+}\right)$ with $F^{-}=F^{+}=\lambda h \in\left\langle X \rightarrow \mathbb{R}^{+}\right\rangle \cdot h(x)$, and for every $f: X \rightarrow T Y$, let $f^{\dagger}\left(F^{-}, F^{+}\right)=\left(\lambda h \in\left\langle Y \rightarrow \mathbb{R}^{+}\right\rangle \cdot F^{-}\left(\lambda x \in X \cdot f^{-}(x)(h)\right), \lambda h \in\left\langle Y \rightarrow \mathbb{R}^{+}\right\rangle\right.$. 
$\left.F^{+}\left(\lambda x \in X \cdot f^{+}(x)(h)\right)\right)$, where by convention $f(x)=\left(f^{-}(x), f^{+}(x)\right)$. Then $(\boldsymbol{T}, \boldsymbol{\eta}, \boldsymbol{\mu})$ is a monad on Top. Together with $\boldsymbol{t}_{X, Y}: X \times \boldsymbol{T} Y \rightarrow \boldsymbol{T}(X \times Y)$ defined by $\boldsymbol{t}_{X, Y}\left(x,\left(F^{-}\right.\right.$, $\left.\left.F^{+}\right)\right)=\left(\lambda h \in\left\langle Y \rightarrow \mathbb{R}^{+}\right\rangle \cdot F^{-}(\lambda y \in Y \cdot h(x, y)), \lambda h \in\left\langle Y \rightarrow \mathbb{R}^{+}\right\rangle \cdot F^{+}(\lambda y \in\right.$ $Y \cdot h(x, y)))$, it forms a strong monad on Cpo and Pcpo.

Proof. That the strong monad laws are satisfied is obvious. The core of the proof is in showing that unit, extension, and tensorial strength are well-defined. We deal with extension. Recall that $f^{\dagger}\left(F^{-}, F^{+}\right)=\left({F^{\prime}}^{-},{F^{\prime}}^{+}\right)$, where ${F^{\prime}}^{-}=\lambda h \in\left\langle Y \rightarrow \mathbb{R}^{+}\right\rangle$. $F^{-}\left(\lambda x \in X \cdot f^{-}(x)(h)\right)$ and $\left.F^{\prime}=\lambda h \in\left\langle Y \rightarrow \mathbb{R}^{+}\right\rangle \cdot F^{+}\left(\lambda x \in X \cdot f^{+}(x)(h)\right)\right)$. Then $F^{\prime-}\left(h+h^{\prime}\right)=\lambda h \in\left\langle Y \rightarrow \mathbb{R}^{+}\right\rangle \cdot F^{-}\left(\lambda x \in X \cdot f^{-}(x)\left(h+h^{\prime}\right)\right) \leq \lambda h \in\langle Y \rightarrow$ $\left.\mathbb{R}^{+}\right\rangle \cdot F^{-}\left(\lambda x \in X \cdot f^{-}(x)(h)+f^{+}(x)\left(h^{\prime}\right)\right)$ (since $f(x)=\left(f^{-}(x), f^{+}(x)\right) \in \mathbf{T Y}$ and $F^{-}$is monotonic $) \leq \lambda h \in\left\langle Y \rightarrow \mathbb{R}^{+}\right\rangle \cdot F^{-}\left(\lambda x \in X \cdot f^{-}(x)(h)\right)+F^{+}(\lambda x \in$ $\left.X \cdot f^{+}(x)\left(h^{\prime}\right)\right)$ (since $\left.\left(F^{-}, F^{+}\right) \in \boldsymbol{T} X\right)={F^{\prime}}^{-}(h)+{F^{\prime}}^{+}\left(h^{\prime}\right)$. Similarly, ${F^{\prime}}^{-}(h)+$ $F^{\prime+}\left(h^{\prime}\right) \leq F^{\prime+}\left(h+h^{\prime}\right)$.

\section{Hearts and Skins}

One of the fundamental theorems of the theory of cooperative games is Shapley's Theorem, which states that every convex game $\nu$ has a non-empty core (on finite discrete $X)$ - the core $\operatorname{Core}(\nu)$ being the set of measures $p$ such that $\nu \leq p$ and $\nu(X)=p(X)$. A refinement of this is Rosenmuller's Theorem, which states that a game $\nu$ is convex iff its core is non-empty and for every function $f: X \rightarrow \mathbb{R}^{+}$, the integral of $f$ along $\nu$ is the minimum of all integrals of $f$ along $p, p \in \operatorname{Core}(\nu)$. In particular, there is a measure $p$ such that $\nu \leq p, \nu(X)=p(X)$, and integrating $f$ along $p$ gives the same result as integrating it along $\nu[5]^{2}$.

We show that the same results hold in the continuous case in [7, chapitre 10]. Remember that games correspond to collinear previsions. Our purpose here is to show that similar theorems hold on previsions that need not be collinear (see [7, chapitre 11] for a more complete development). The analogue of measures will be linear previsions. We drop the analogue of the $\nu(X)=p(X)$ condition, however we concentrate on normalized games and previsions, because the technical treatment is slightly easier. We call the analogue of cores hearts, and the dual notion skin.

Definition 4 (Heart, Skin). For any function $F$ from $\left\langle X \rightarrow \mathbb{R}^{+}\right\rangle$to $\mathbb{R}^{+}$, its heart Coeur $(F)$ is the set of linear functionals $G$ such that $F \leq G$. Its continuous heart $C C$ Cour $(F)$ is the subset of those Gs that are continuous. Its skin Peau $(F)$ is the set of linear functionals $G$ such that $G \leq F$. Its continuous skin $C P e a u(F)$ is the subset of those functionals $G$ that are continuous.

\footnotetext{
${ }^{2}$ An anonymous referee for a previous version of this paper asked whether this had anything to do with a theorem due to Shannon, stating that for any distribution $p$ and function $f$, there is another distribution $p^{\prime}$ such that $f$ has the same mean relative to $p$ and $p^{\prime}$, and which maximizes entropy. While there is a similar flavor to it, I must confess that I don't see any relationship. Moreover, Shannon's Theorem, contrarily to Rosenmuller's, does not extend to the continuous case, if only because entropy is only defined on finite spaces (relative entropy is another matter). I won't include this footnote in the final version of this paper.
} 
Again, we let $\operatorname{Coeur}_{1}(F)$, CCoeur $_{1}(F), \ldots$, be the subsets of the corresponding spaces consisting of normalized previsions only, and similarly $\operatorname{Coeur}_{\leq 1}(F), \ldots$, for those consisting of sub-normalized previsions.

Most of the developments below rest on Roth's Sandwich Theorem ([21], [24, Theorem 3.1]), which states that on every ordered cone $C$, for every positively homogeneous super-additive function $q: C \rightarrow \overline{\mathbb{R}}^{+}$and every positively homogeneous sub-additive function $p: C \rightarrow \overline{\mathbb{R}}^{+}$such that $a \leq b$ implies $q(a) \leq p(b)$ (e.g., when $q \leq p$ and either $q$ or $p$ is monotonic), then there is a monotonic linear function $f: C \rightarrow \overline{\mathbb{R}}^{+}$ such that $q \leq f \leq p . \overline{\mathbb{R}}^{+}$is $\mathbb{R}^{+}$plus an extra point at infinity $+\infty$. A cone is a set $C$, together with a binary operation + turning it into a commutative monoid and a scalar multiplication $\cdot$ from $\mathbb{R}^{+} \times C$ to $C$, such that $1 \cdot a=a, 0 \cdot a=0,(r s) \cdot a=r \cdot(s \cdot a)$, $r \cdot(a+b)=r \cdot a+r \cdot b$, and $(r+s) \cdot a=r \cdot a+s \cdot a$. An ordered cone is equipped in addition with a partial ordering $\leq$ making + and $\cdot$ monotonic. We only use Roth's Theorem on ordered cones of the form $\left\langle X \rightarrow \mathbb{R}^{+}\right\rangle$. Our key result is:

Theorem 3. Let $X$ be a stably locally compact space, $F$ a continuous lower prevision, and $f$ a bounded continuous function from $X$ to $\mathbb{R}^{+}$. Then there is a continuous linear functional $G$ from $\left\langle X \rightarrow \mathbb{R}^{+}\right\rangle$to $\overline{\mathbb{R}}^{+}$such that $F \leq G$ and $F(f)=G(f)$.

Proof. Let $F$ be a lower prevision on $X$, and $f \in\left\langle X \rightarrow \mathbb{R}^{+}\right\rangle$. Define $\breve{F}_{f}$ by $\breve{F}_{f}(g)=$ $\inf _{\substack{\lambda \in \mathbb{R}^{+} \\ \lambda f \geq g}}\left[F(\lambda f)-\sup _{\substack{h \in\left\langle X \rightarrow \mathbb{R}^{+}\right\rangle \\ g+h \leq \lambda f}} F(h)\right]$, taking this to be $+\infty$ is there is no $\lambda \in \mathbb{R}^{+}$ such that $\lambda f \geq g$. One checks that $\widetilde{F}_{f}$ is monotonic, positively homogeneous, subadditive, above $F\left(\breve{F}_{f}(g) \geq F(g)\right.$ for all $\left.g\right)$, touches $F$ at $f\left(\breve{F}_{f}(f)=F(f)\right)$. Apply Roth's Sandwich Theorem gives us a monotonic linear functional $G_{0}$ such that $F \leq G_{0}$ and $F(f)=G_{0}(f)$. However, $G_{0}$ may fail to be continuous. One now observes that $\left\langle X \rightarrow \mathbb{R}^{+}\right\rangle$is a continuous poset, with a basis $B$ consisting of step functions. By Scott's Formula, the functional $G$ defined by $G(f)=\sup _{g \in B, g \ll f} G_{0}(g)$ is continuous; in fact, the largest continuous functional below $G_{0}$. It follows that $F \leq G$ and $F(f)=G(f)$. The most difficult part of the proof is showing that $G$ is linear. This rests on the fact that $\ll$ is multiplicative i.e., for any $a>0, f \ll g$ iff $a \cdot f \ll a \cdot g$, and additive, i.e., if $h, f, g \in\left\langle X \rightarrow \mathbb{R}^{+}\right\rangle$are such that $h \ll f+g$, then $h \leq f^{\prime}+g^{\prime}$ for some $f^{\prime}, g^{\prime} \in B$ with $f^{\prime} \ll f, g^{\prime} \ll g$; and conversely, $f^{\prime} \ll f$ and $g^{\prime} \ll g$ imply $f^{\prime}+g^{\prime} \ll f+g$.

Note that $G$ may take the value $+\infty$. We can refine this in the case of normalized previsions (for sub-normalized previsions, see [7, section 11.4]):

Theorem 4. Let $X$ be a stably locally compact space, $F$ a normalized continuous lower prevision on $X$, and $f$ a bounded continuous function from $X$ to $\mathbb{R}^{+}$. Then there is a normalized continuous linear prevision $G$ such that $F \leq G$ and $F(f)=G(f)$.

Proof. Similar to Theorem 3. However, it may be that $\breve{F}_{f}$ reaches $+\infty$. Refine this by letting $\breve{F}_{f}(g)=\inf _{\epsilon \in \mathbb{R}^{+}} \breve{F}_{f+\epsilon}(g)$, and using $\breve{F}_{f}$ instead of $\breve{F}_{f}$. One checks that, since $F$ is normalized, $\breve{F}_{f+\epsilon}$ is antitone in $\epsilon$. Then $\breve{F}_{f}$ is again monotonic, positively homogeneous, sub-additive (using antitony in $\epsilon$ ), above $F$, and touches $F$ at $f$. Moreover, 
it is easy to see that $\breve{F}_{f}\left(\chi_{X}\right)=1$. We build $G_{0}$, then $G$ from $\breve{F}_{f}$, as in Theorem 3 . Additionally, we need $X$ to be compact so as to establish that $G\left(\chi_{X}\right)=1$. Since $G$ is linear, it follows that $G$ is normalized.

One can deal with upper previsions instead, see [7, section 11.5], using a notion we call convex-concave duality to reduce to the above. We then obtain [7, théorème 11.5.22] that, when $X$ is stably compact, $F$ is a normalized continuous upper prevision on $X$, there is a normalized continuous linear prevision $G$ on $X$ such that $G \leq F$. Moreover, for every $f \in\left\langle X \rightarrow \mathbb{R}^{+}\right\rangle, F(f)=\sup _{G \in C P \text { eau }}(F) G(f)$.

Theorem 4 allows us to state a form of Rosenmuller's Theorem:

Theorem 5. Let $X$ be stably locally compact, $F$ a continuous normalized prevision on $X$. Then $F$ is lower iff $C$ Coeur $_{1}(F) \neq \emptyset$ and for every $f \in\left\langle X \rightarrow \mathbb{R}^{+}\right\rangle, F(f)=$ $\inf _{G \in C_{C \text { Cour }}(F)} G(f)$. In this case, the inf is attained: $F(f)=\min _{G \in C C_{\text {Cour }}(F)} G(f)$.

There is, of course, a dual theorem on upper previsions and their skins [7, théorème 11.7.4]; infs are replaced by sups, which need not be attained.

To go further, we need to consider another topology on spaces of previsions: the weak topology is the coarsest that makes the function $F \mapsto F(f)$ continuous, for each $f \in\left\langle X \rightarrow \mathbb{R}^{+}\right\rangle$. The Scott topology is in general finer. Write $\nabla \mathbf{P}_{1 w k}(X)$ the space $\nabla \mathbf{P}_{1}(X)$ with the weak topology, and similarly for other spaces. Then:

Proposition 4. Let $X$ be stably compact, $F$ a normalized continuous lower prevision, then $C$ Coeur $r_{1}(F)$ is a non-empty saturated compact convex subset of $\mathbf{P}_{1 \text { wk }}^{\triangle}(X)$.

Compactness can be deduced from Plotkin's version of the Banach-Alaoglu Theorem [19], while convexity (i.e., $\alpha F+(1-\alpha) F^{\prime}$ is in $C_{C o e u r}(F)$ as soon as $F$ and $F^{\prime}$ are, $\alpha \in[0,1])$ is clear. It is much easier to show that the continuous skin $\operatorname{CPeau}_{1}(F)$ of a normalized continuous upper prevision $F$ is closed:

Proposition 5. Let $X$ be a topological space, $F$ a normalized continuous upper prevision, then $C \operatorname{Peau}_{1}(F)$ is a closed convex subset of $\mathbf{P}_{1 \text { wk }}^{\triangle}(X)$. It is non-empty as soon as $X$ is stably compact.

Finally, call a lens of a space $X$ any non-empty intersection $L=Q \cap F$ of a saturated compact $Q$ and a closed subset $F$. Then:

Proposition 6. Let $X$ be a stably compact space. The continuous normalized body $C_{C o r p s}(F)=C_{1} C_{\text {oeur }}\left(F^{-}\right) \cap C$ Peau $_{1}\left(F^{+}\right)$of a continuous normalized fork $F=\left(F^{-}, F^{+}\right)$on $X$ is a lens. Moreover, $C_{C o e u r}\left(F^{-}\right)=\uparrow C C C o r p s_{1}(F)$ and $\operatorname{CPeau}_{1}\left(F^{+}\right)=\downarrow \operatorname{CCorps}_{1}(F)$.

Proof. We show that: $(*)$ whenever $G \in \operatorname{CCoeur}_{1}\left(F^{-}\right)$, there is some $G^{\prime} \in C$ Coeur $_{1}\left(F^{-}\right) \cap$ $C \operatorname{Peau}_{1}\left(F^{+}\right)$such that $G^{\prime} \leq G$. Let $F^{\prime}(h)=\inf _{\substack{f, g \in\left\langle X \rightarrow \mathbb{R}^{+}\right\rangle \\ f+g \geq h}}\left(F^{+}(f)+G(g)\right)$. One checks that $F^{-} \leq F^{\prime} \leq G$, that $F^{\prime}$ is an upper prevision, so by Roth's Sandwich Theorem, there is a linear monotonic functional $G_{0}$ such that $F^{-} \leq G_{0} \leq F^{\prime}$. Since $G_{0} \leq F^{\prime}, G_{0}$ does not take the value $+\infty$. Build $G$ from $G_{0}$ using Scott's Formula, as before. It is easy to see that $G$ is a continuous, normalized, linear prevision. Since 
$F^{-} \leq G^{\prime}, G^{\prime} \in C_{\text {Coeur }}\left(F^{-}\right)$. Since $G^{\prime} \leq F^{\prime} \leq F^{+}, G^{\prime} \in \operatorname{CPeau}_{1}\left(F^{+}\right)$. Since $F^{\prime} \leq F^{\prime} \leq G, G^{\prime} \leq G$.

By $(*), C_{C o e u r}\left(F^{-}\right) \cap C_{\text {Peau }}\left(F^{+}\right)$is non-empty. That $C$ Coeur $_{1}\left(F^{-}\right)=$ $\uparrow\left(C\right.$ Coeur $\left._{1}\left(F^{-}\right) \cap C \operatorname{Peau}_{1}\left(F^{+}\right)\right)$is another easy consequence of $(*)$. That $C \operatorname{Peau}_{1}\left(F^{+}\right)=$ $\downarrow\left(C_{\text {Coeur }}\left(F^{-}\right) \cap C\right.$ Peau $\left._{1}\left(F^{+}\right)\right)$can be shown in a similar way, by defining $F^{\prime \prime}(h)=$ $\sup _{f, g \in\left\langle X \rightarrow \mathbb{R}^{+}\right\rangle}\left(F^{-}(f)+G(g)\right)$, where $G \in C P e a u_{1}\left(F^{-}\right)$, and using $F^{\prime \prime}$ to show that there is some $G^{\prime} \in C_{C o e u r}\left(F^{-}\right) \cap C \operatorname{Peau}_{1}\left(F^{+}\right)$such that $G \leq G^{\prime}$.

The last three propositions state that any normalized continuous lower prevision, resp. upper prevision, resp. fork $F$ gives rise to an element $C$ Coeur $1(F)$, resp. $C \operatorname{Peau}_{1}(F)$, resp. $C \operatorname{Corps}_{1}(F)$ of the Smyth powerdomain $\mathcal{Q}\left(\mathbf{P}_{1 w k}^{\triangle}(X)\right)$ (demonic non-deterministic choice of a probability distribution-remember that $\left.\mathbf{P}_{1}^{\triangle}(X) \cong \mathbf{V}_{1}(X)\right)$, resp. the Hoare powerdomain $\mathcal{H}\left(\mathbf{P}_{1 w k}^{\triangle}(X)\right)$ over $\mathbf{P}_{1 w k}^{\triangle}(X)$ (angelic), resp. the Plotkin powerdomain over $\mathbf{P}_{1 w k}^{\triangle}(X)$ (chaotic). This is a form of completeness: our spaces of previsions and of forks contain no junk, and really are no more than mixes of nondeterministic and probabilistic choice.

In the converse direction, still assuming $X$ stably compact, there is a map $\Pi$ : $\mathcal{Q}\left(\mathbf{P}_{1 \text { wk }}^{\triangle}(X)\right) \rightarrow \nabla \mathbf{P}_{1}(X)$ defined by $\Pi K(f)=\min _{G \in K} G(f)$, and $C$ Coeur 1 $\Pi$ is a Galois injection consisting of continuous maps [7, théorème 11.7.10], while there is a continuous map $\bigsqcup: \mathcal{H}\left(\mathbf{P}_{1 w k}^{\triangle}(X)\right) \rightarrow \nabla \mathbf{P}_{1}(X)$ defined by $\bigsqcup C(f)=$ $\sup _{G \in C} G(f)$, so that $\bigsqcup \dashv C P e a u_{1}$ is a Galois surjection.

We conclude by noticing that, when $X$ is a continuous cpo with a least element, $\mathbf{P}_{1 w k}^{\triangle}(X)$ is homeomorphic to $\mathbf{V}_{1}(X)$ with the weak topology, and the latter coincides then with the Scott topology [9]. Apart from spurious details (e.g., we bound our valuations by 1 instead of $+\infty$ ), there is therefore a strong connection with the models of Mislove [16] and Tix [23,24]. The question whether the Galois connections above can be turned into isomorphisms remains open.

\section{References}

1. Samson Abramsky and Achim Jung. Domain theory. In S. Abramsky, D. M. Gabbay, and T. S. E. Maibaum, editors, Handbook of Logic in Computer Science, volume 3, pages 1-168. Oxford University Press, 1994.

2. Martín Escardó and Reinhold Heckmann. Topologies on spaces of continuous functions. Topology Proceedings, 26(2):545-564, 2001-2002.

3. Neil Ghani and Tarmo Uustalu. Coproducts of ideal monads. Theoretical Informatics and Applications, 38(4):321-342, 2004. Extended abstract in Z. Ésik, I. Walukiewicz, ed., Proc. of Int. Workshop on Fixed Points in Computer Science, FICS'03 (Warsaw, Apr. 2003), pp. 32-36. Warsaw Univ., 2003.

4. Gerhard Gierz, Karl Heinrich Hofmann, Klaus Keimel, Jimmie D. Lawson, Michael Mislove, and Dana S. Scott. A Compendium of Continuous Lattices. Springer Verlag, 1980.

5. Itzhak Gilboa and David Schmeidler. Additive representation of non-additive measures and the Choquet integral. Discussion Papers 985, Northwestern University, Center for Mathematical Studies in Economics and Management Science, 1992.

6. Jean Goubault-Larrecq. Continuous capacities on continuous state spaces. In ICALP'2007. Springer-Verlag LNCS, 2007. 
7. Jean Goubault-Larrecq. Une introduction aux capacités, aux jeux et aux prévisions. http://www.lsv.ens-cachan.fr/ goubault/ProNobis/pp.pdf, January 2007. 516 pages.

8. Claire Jones. Probabilistic Non-Determinism. PhD thesis, University of Edinburgh, 1990. Technical Report ECS-LFCS-90-105.

9. Achim Jung. Stably compact spaces and the probabilistic powerspace construction. In J. Desharnais and P. Panangaden, editors, Domain-theoretic Methods in Probabilistic Processes, volume 87 of Electronic Lecture Notes in Computer Science. Elsevier, 2004. 15pp.

10. G. Max Kelly. A unified treatment of transfinite constructions for free algebras, free monoids, colimits, associated sheaves and so on. Bulletin of the Australian Mathematical Society, 22:1-83, 1980.

11. Christoph Lüth. Categorical Term Rewriting: Monads and Modularity. PhD thesis, University of Edinburgh, 1997.

12. Sebastian Maßß. Coherent lower previsions as exact functionals and their (sigma-)core. In Gert De Cooman, Terrence Fine, and Teddy Seidenfeld, editors, Proceedings of the 2nd Intl. Symp. Imprecise Probabilities and their Applications (ISIPTA'01), pages 230-236, Ithaca, NY, 2001.

13. Sebastian Maass. Continuous linear representation of coherent lower previsions. In JeanMarc Bernard, Teddy Seidenfeld, and Marco Zaffalon, editors, Proc. 3rd Intl. Symp. on Imprecise Probabilities and Their Applications (ISIPTA'03), pages 371-381, Lugano, Switzerland, July 2003. Carleton Scientific Proceedings in Informatics 18.

14. Ernest G. Manes. Algebraic Theories, volume 26 of Graduate Texts in Mathematics. Springer-Verlag, 1976.

15. Michael Mislove. Topology, domain theory and theoretical computer science. Topology and Its Applications, 89:3-59, 1998.

16. Michael Mislove. Nondeterminism and probabilistic choice: Obeying the law. In Proc. 11th Conf. Concurrency Theory (CONCUR'00), pages 350-364. Springer Verlag LNCS 1877, 2000 .

17. Eugenio Moggi. Notions of computation and monads. Information and Computation, 93:5592, 1991.

18. Martin J. Osborne and Ariel Rubinstein. A Course in Game Theory. MIT Press, 1994.

19. Gordon Plotkin. A domain-theoretic Banach-Alaoglu theorem. Mathematical Structures in Computer Science, 16:299-311, 2006.

20. Norman Ramsey and Avi Pfeffer. Stochastic lambda calculus and monads of probability distributions. In Proceedings of the 29th Annual ACM SIGPLAN-SIGACT Symposium on Principles of Programming Languages (POPL'02), pages 154-165, 2002.

21. Walter Roth. Hahn-Banach type theorems for locally convex cones. Journal of the Australian Mathematical Society, 68(1):104-125, 2000.

22. Regina Tix. Stetige Bewertungen auf topologischen Räumen. Diplomarbeit, TH Darmstadt, June 1995.

23. Regina Tix. Continuous D-Cones: Convexity and Powerdomain Constructions. PhD thesis, Technische Universität Darmstadt, 1999.

24. Regina Tix, Klaus Keimel, and Gordon Plotkin. Semantic domains for combining probability and non-determinism. Electronic Notes in Theoretical Computer Science, 129:1-104, 2005.

25. Daniele Varacca. The powerdomain of indexed valuations. In Proc. 17th Annual IEEE Symp. on Logic in Computer Science (LICS'02), pages 299-308, Copenhagen, July 2002. IEEE Computer Society Press.

26. Philip Wadler. Comprehending monads. Mathematical Structures in Computer Science, 2:461-493, 1992.

27. Peter Walley. Statistical Reasoning with Imprecise Probabilities. Chapman and Hall, London, 1991. 


\section{A Proofs of Theorems}

Theorem 1. $\alpha_{e} \dashv \gamma_{\mathcal{e}}$ is a Galois injection from (plain, convex, concave) games into (plain, lower, upper) collinear previsions. That is, $\alpha_{\mathrm{e}}$ and $\gamma_{\mathrm{e}}$ are monotonic, $\alpha_{\mathrm{e}}\left(\gamma_{\mathrm{e}}(F)\right) \leq$ $F$ for every collinear prevision $F$, and $\gamma_{\mathfrak{e}}\left(\alpha_{\mathfrak{C}}(\nu)\right)=\nu$ for every game $\nu$.

Moreover, when restricted to continuous previsions and games, $\alpha_{\mathrm{e}}$ and $\gamma_{\mathrm{e}}$ define an isomorphism between $\mathbf{J}(X)$ and $\mathbf{P}^{*}(X)$, between $\nabla \mathbf{J}(X)$ and $\nabla \mathbf{P}^{*}(X)$, between $\triangle \mathbf{J}(X)$ and $\triangle \mathbf{P}^{*}(X)$.

Proof. Let $F$ be a prevision. Then $\gamma_{\mathfrak{C}}(F)(\emptyset)=0$ because $F$ is positively homogeneous (take $\alpha=0$ ); $\gamma_{\mathfrak{e}}(F)$ is monotonic because $F$ is. So $\gamma_{\mathfrak{e}}(F)$ is a game.

If $F$ is a lower prevision, then notice that $\chi_{U \cup V}$ and $\chi_{U \cap V}$ are comonotonic: assume $\chi_{U \cup V}(x)<\chi_{U \cup V}\left(x^{\prime}\right)$ and $\chi_{U \cap V}(x)>\chi_{U \cap V}\left(x^{\prime}\right)$, then necessarily $x \notin U \cup V$ and $x \in U \cap V$, which is impossible. Next, note that $\chi_{U \cup V}+\chi_{U \cap V}=\chi_{U}+\chi_{V}$. So $\gamma_{\mathcal{e}}(F)(U \cup V)+\gamma_{\mathcal{e}}(F)(U \cap V)=F\left(\chi_{U \cup V}+\chi_{U \cap V}\right)$ (since $F$ is collinear) $=$ $F\left(\chi_{U}+\chi_{V}\right) \geq F\left(\chi_{U}\right)+F\left(\chi_{V}\right)$ (since $F$ is super-additive) $=\gamma_{\mathrm{e}}(F)(U)+\gamma_{\mathrm{e}}(F)(V)$. Similarly, $\gamma_{\mathfrak{e}}(F)$ is concave if $F$ is upper.

In the sequel, we shall need the following claim.

Claim A. Let $F$ be a collinear prevision on $X$, and $f=a+\sum_{i=1}^{m} a_{i} \chi_{U_{i}}$ a step function, with $U_{1} \supseteq \ldots \supseteq U_{m}, a \in \mathbb{R}, a_{1}, \ldots, a_{m} \in \mathbb{R}^{+}$. Then:

$$
\oint_{x \in X} f(x) d \gamma_{\mathcal{C}}(F)=F(f)
$$

Proof. Let $U_{0}=X$ and $a_{0}=a$, to make notation uniform. Then:

$$
\begin{aligned}
\oint_{x \in X} f(x) d \gamma_{\mathfrak{e}}(F) & =a \gamma_{\mathfrak{e}}(F)(X)+\sum_{i=1}^{m} a_{i} \gamma_{\mathfrak{e}}(F)\left(U_{i}\right) \\
& =a F\left(\chi_{X}\right)+\sum_{i=1}^{m} a_{i} F\left(\chi_{U_{i}}\right)=F\left(a \chi_{X}\right)+\sum_{i=1}^{m} F\left(a_{i} \chi_{U_{i}}\right) \\
& =\sum_{i=0}^{m} F\left(a_{i} \chi_{U_{i}}\right)
\end{aligned}
$$

Note that $U_{0} \supseteq U_{1} \supseteq \ldots \supseteq U_{m}$. Wlog., assume that $a_{1}, \ldots, a_{m}>0$. For each $k, 1 \leq k \leq m$, the functions $\sum_{i=0}^{k-1} a_{i} \chi_{U_{i}}$ and $a_{k} \chi_{U_{k}}$ are comonotonic. Indeed, assume $\left(\sum_{i=0}^{k-1} a_{i} \chi_{U_{i}}\right)(x)>\left(\sum_{i=0}^{k-1} a_{i} \chi_{U_{i}}\right)\left(x^{\prime}\right)$ and $a_{k} \chi_{U_{k}}(x)<a_{k} \chi_{U_{k}}\left(x^{\prime}\right)$. The latter implies that $x \notin U_{k}$, and $x^{\prime} \in U_{k}$. Since $U_{0} \supseteq U_{1} \supseteq \ldots \supseteq U_{m}, x^{\prime}$ is in every $U_{i}, 0 \leq i \leq k-1$, so $\left(\sum_{i=0}^{k-1} a_{i} \chi_{U_{i}}\right)\left(x^{\prime}\right)=\sum_{i=0}^{k-1} a_{i} \geq\left(\sum_{i=0}^{\bar{k}-1} a_{i} \chi_{U_{i}}\right)(x)$, a contradiction.

Since $F$ is collinear, it follows:

$$
\sum_{i=0}^{m} F\left(a_{i} \chi_{U_{i}}\right)=F\left(\sum_{i=0}^{k-1} a_{i} \chi_{U_{i}}\right)+\sum_{i=k}^{m} F\left(a_{i} \chi_{U_{i}}\right)
$$


for every $k, 1 \leq k \leq m+1$, by induction on $k$. So, for $k=m+1$,

$$
\oint_{x \in X} f(x) d \gamma_{\mathcal{e}}(F)=F\left(\sum_{i=0}^{m} a_{i} \chi_{U_{i}}\right)=F(f)
$$

We now show that $\gamma_{\mathfrak{e}}\left(\alpha_{\mathfrak{e}}(\nu)\right)=\nu$ for every game $\nu$ :

$$
\gamma_{\mathcal{e}}\left(\alpha_{\mathcal{e}}(\nu)\right)(U)=\alpha_{\mathcal{E}}(\nu)\left(\chi_{U}\right)=\oint_{x \in X} \chi_{U}(x) d \nu=\nu(U)
$$

Once we know $\alpha_{\mathcal{e}} \dashv \gamma_{\mathcal{e}}$ is a Galois connection, this will imply it is a Galois injection. To show that $\alpha_{\mathcal{e}} \dashv \gamma_{\mathcal{e}}$ is a Galois connection, it remains to show that $\alpha_{\mathcal{e}}$ is monotonic (which is clear), that $\gamma_{\mathcal{e}}$ is monotonic (clear since previsions are monotonic), and that $\alpha_{\mathcal{e}}\left(\gamma_{\mathcal{e}}(F)\right) \leq F$ for every collinear prevision $F$. First,

$$
\alpha_{\mathcal{e}}\left(\gamma_{\mathcal{e}}(F)\right)(f)=\oint_{x \in X} f(x) d \gamma_{\mathcal{C}}(F)
$$

Using the step functions $f_{K}, K \in \mathbb{N}$, by Claim A:

$$
\oint_{x \in X} f_{K}(x) d \gamma_{\mathcal{e}}(F)=F\left(f_{K}\right)
$$

The least upper bound of the left-hand side is the Choquet integral of $f$ along $\gamma_{\mathfrak{e}}(F)$, i.e., $\alpha_{\mathcal{C}}\left(\gamma_{\mathcal{C}}(F)\right)(f)$. Since $F$ is monotonic, the right-hand side is less than or equal to $F(f)$, so $\alpha_{\mathcal{e}}\left(\gamma_{\mathcal{e}}(F)\right)(f) \leq F(f)$.

Let us turn to continuous games and continuous previsions. If $\nu$ is a continuous game, then $\alpha_{\mathcal{e}}(\nu)$ is continuous, since Choquet integration is Scott-continuous in its function argument. Conversely, if $F$ is continuous, then for every directed family $\left(U_{i}\right)_{i \in I}$ of opens, $\gamma_{\mathcal{C}}(F)\left(\bigcup_{i \in I} U_{i}\right)=F\left(\chi_{\bigcup_{i \in I} U_{i}}\right)=F\left(\sup _{i \in I} \chi_{U_{i}}\right)=\sup _{i \in I} F\left(\chi_{U_{i}}\right)=$ $\sup _{i \in I} \gamma_{\mathfrak{e}}(F)\left(U_{i}\right)$, so $\gamma_{\mathfrak{e}}(F)$ is continuous. Now if $F$ is continuous, then the least upper bound of the right-hand side of (4) is $F(f)$, since $f=\sup _{K \in \mathbb{N}} f_{K}$ and $F$ is continuous; while the left-hand side is the Choquet integral of $f$ along $\gamma_{\mathfrak{C}}(F)$, i.e., $\alpha_{\mathfrak{e}}\left(\gamma_{\mathcal{e}}(F)\right)(f)$. So $\alpha_{\mathfrak{e}}\left(\gamma_{\mathcal{C}}(F)\right)(f)=F(f)$, whence the isomorphism.

Theorem 2. Define $\boldsymbol{T} X$ as $\mathbf{P}(X)$, resp. $\nabla \mathbf{P}(X)$, resp. $\triangle \mathbf{P}(X)$. Let $\boldsymbol{\eta}_{X}(x)=$ $\lambda h \in\left\langle X \rightarrow \mathbb{R}^{+}\right\rangle \cdot h(x)$, and $f^{\dagger}(F)(h)=F(\lambda x \in X \cdot f(x)(h))$ for every $f: X \rightarrow T Y$. Then $\boldsymbol{T}$ is a monad on Top, i.e., $\left(\boldsymbol{T}, \boldsymbol{\eta},{ }_{-}^{\dagger}\right)$ is a Kleisli triple. On Posc, $\boldsymbol{T}$ is a strong monad: $\boldsymbol{t}_{X, Y}: X \times \boldsymbol{T} Y \rightarrow \boldsymbol{T}(X \times Y)$ defined as $\boldsymbol{t}_{X, Y}(x, F)(h)=F(\lambda y \in Y \cdot h(x, y))$ is a tensorial strength.

Proof. We must first show that, for every $f: X \rightarrow T Y, f^{\dagger}$ is indeed a continuous map from $\boldsymbol{T} X$ to $\boldsymbol{T} Y$. Foremost, we must make sure that for every continuous (plain, lower, upper) prevision $F$ on $X, f^{\dagger}(F)$ is a continuous (plain, lower, upper) prevision on $Y$. Positive homogeneity: $f^{\dagger}(F)(\alpha h)=F(\lambda x \in X \cdot f(x)(\alpha h))=F(\lambda x \in X \cdot \alpha f(x)(h))$ (since $f(x) \in T Y$ is positively homogeneous) $=F(\alpha \lambda x \in X \cdot f(x)(h))=\alpha F(\lambda x \in$ $X \cdot f(x)(h))$ (since $F \in T X$ is positively homogeneous) $=\alpha f^{\dagger} F(h)$. Monotonicity: 
assume $h \leq h^{\prime}$, then for each $x \in X, f(x)(h) \leq f(x)\left(h^{\prime}\right)$ since $f(x) \in T Y$ is monotonic, so $f^{\dagger}(F)(h)=F(\lambda x \in X \cdot f(x)(h)) \leq F\left(\lambda x \in X \cdot f(x)\left(h^{\prime}\right)\right)=$ $f^{\dagger}(F)\left(h^{\prime}\right)$, since $F \in \boldsymbol{T} X$ is monotonic. In the case $\boldsymbol{T}=\nabla \mathbf{P}, F$ and every $f(x)$ is super-additive, so $f^{\dagger}(F)\left(h+h^{\prime}\right)=F\left(\lambda x \in X \cdot f(x)\left(h+h^{\prime}\right)\right) \geq F(\lambda x \in X$. $\left.f(x)(h)+f(x)\left(h^{\prime}\right)\right) \geq F(\lambda x \in X \cdot f(x)(h))+F\left(\lambda x \in X \cdot f(x)\left(h^{\prime}\right)\right)=f^{\dagger}(F)(h)+$ $f^{\dagger}(F)\left(h^{\prime}\right)$, so $f^{\dagger} F$ is super-additive, too. Similarly when $\boldsymbol{T}=\triangle \mathbf{P}$. Continuity: let $\left(h_{i}\right)_{i \in I}$ be a directed family of bounded continuous functions from $Y$ to $\mathbb{R}^{+}$with $h$ as least upper bound. Then $f^{\dagger}(F)\left(\sup _{i \in I} h_{i}\right)=F\left(\lambda x \cdot f(x)\left(\sup _{i \in I} h_{i}\right)\right)=F(\lambda x$. $\left.\sup _{i \in I} f(x)\left(h_{i}\right)\right)$ (since $f(x) \in \boldsymbol{T} Y$ is continuous) $=\sup _{i \in I} F\left(\lambda x \cdot f(x)\left(h_{i}\right)\right)$ (since $F \in \boldsymbol{T} X$ is continuous).

Then we must show that $f^{\dagger}$ is continuous. Since $T X$ and $T Y$ are posets with the Scott topology, it is enough to show that for any directed family $\left(F_{i}\right)_{i \in I}$ in $T X$, $f^{\dagger}\left(\sup _{i \in I} F_{i}\right)=\sup _{i \in I} f^{\dagger}\left(F_{i}\right)$. But this is obvious from the definition.

We now check the Kleisli triple axioms. This is in fact automatic, since $\boldsymbol{T}$ is defined as a continuation-style monad. (1) $\boldsymbol{\eta}_{X}^{\dagger}(F)(h)=F\left(\lambda x \in X \cdot \boldsymbol{\eta}_{X}(x)(h)\right)=F(\lambda x \in$ $X \cdot h(x))=F(h)$, so $\boldsymbol{\eta}_{X}^{\dagger}=\operatorname{id}_{X}$. (2) Let $f: X \rightarrow \boldsymbol{T} Y$, then $\left(f^{\dagger} \circ \boldsymbol{\eta}_{X}\right)(x)(h)=$ $f^{\dagger}\left(\boldsymbol{\eta}_{X}(x)\right)(h)=\boldsymbol{\eta}_{X}(x)\left(\lambda x^{\prime} \in X \cdot f\left(x^{\prime}\right)(h)\right)=f(x)(h)$, so $f^{\dagger} \circ \boldsymbol{\eta}_{X}=f$. (3) Let $g: X \rightarrow T Y, f: Y \rightarrow T Z$. On the one hand, $\left(f^{\dagger} \circ g^{\dagger}\right)(F)(h)=f^{\dagger}\left(g^{\dagger}(F)\right)(h)=$ $g^{\dagger}(F)(\lambda y \in Y \cdot f(y)(h))=F(\lambda x \in X \cdot g(x)(\lambda y \in Y \cdot f(y)(h)))$. On the other hand, $\left(f^{\dagger} \circ g\right)^{\dagger}(F)(h)=F\left(\lambda x \in X \cdot\left(f^{\dagger} \circ g\right)(x)(h)\right)=F\left(\lambda x \in X \cdot f^{\dagger}(g(x))(h)\right)=$ $F(\lambda x \in X \cdot g(x)(\lambda y \in Y \cdot f(y)(h)))$, whence $f^{\dagger} \circ g^{\dagger}=\left(f^{\dagger} \circ g\right)^{\dagger}$.

Contrarily to what might be expected, $\boldsymbol{t}_{X, Y}$ is not defined on all of $\boldsymbol{T o p}$. The reason is that it may fail to be continuous. On Posc, this is repaired by the fact that a function of two arguments is continuous iff it is continuous in each argument separately (a fact that fails in Top). Let us be more precise. Let $h$ be any bounded continuous function from $X \times Y$ to $\mathbb{R}^{+}$. For any fixed $x$, the function $\lambda y \in Y \cdot h(x, y)$ is bounded and continuous, so $F(\lambda y \in Y \cdot h(x, y))$ makes sense. It is clear that the function $\boldsymbol{t}_{X, Y}(x, F)$ mapping $h \in\left\langle X \times Y \rightarrow \mathbb{R}^{+}\right\rangle$to $F(\lambda y \in Y \cdot h(x, y))$ is a continuous (plain, lower, upper) prevision, since $F$ is. Now $\boldsymbol{t}_{X, Y}(x, F)$ is obviously Scott-continuous in $F$ ( $x$ fixed), and also in $x \in X$ ( $F$ fixed), since $h$ is continuous, and $F$ is continuous. So $t_{X, Y}$ is a morphism in Posc.

We need to check the tensorial strength equations [17]. These are in fact obvious, as again $\boldsymbol{t}_{X, Y}$ is defined exactly as for the continuation monad.

Proposition 1. Theorem 1 again holds for normalized (continuous) games and previsions, and for sub-normalized (continuous) games and previsions.

Proof. It is enough to show that $\alpha_{\mathcal{e}}$ maps normalized (resp. sub-normalized) games to normalized (resp. sub-normalized) previsions, and that $\gamma_{e}$ goes the other way around. The essential point is that: $(*)$ Choquet integration is linear on comonotonic functions, and any constant $a$ is comonotonic with any function $f$. Now if $\nu$ is normalized, i.e., 
$\nu(X)=1$, then:

$$
\begin{aligned}
\alpha_{\mathcal{C}}(\nu)(a+f) & =\oint_{x \in X} a+f(x) d \nu \\
& =\oint_{x \in X} a d \nu+\oint_{x \in X} f(x) d \nu \quad \text { by }(*) \\
& =a+\alpha_{\mathcal{C}}(\nu)
\end{aligned}
$$

When $\nu$ is sub-normalized, the last line is an inequality $\leq$ instead.

Conversely, if $F$ is a normalized prevision, then $\gamma_{\mathfrak{e}}(F)(X)=F\left(\chi_{X}\right)=F(1+f)$ where $f$ is the zero function. Since $F$ is normalized, this equals $1+F(f)=1$, since $F(f)=0$ by positive homogeneity with $\alpha=0$. Similarly, if $F$ is sub-normalized, then $\gamma_{\mathcal{e}}(F)(X)=F(1+f) \leq 1+F(f)=1$.

Proposition 3. Let $\mathbf{T} X$ be defined as $\mathbf{F}(X), \mathbf{F}_{\leq 1}(X)$, or $\mathbf{F}_{1}(X)$. Let $\boldsymbol{\eta}_{X}(x)=$ $\left(F^{-}, F^{+}\right)$with $F^{-}=F^{+}=\lambda h \in\left\langle X \rightarrow \mathbb{R}^{+}\right\rangle \cdot h(x)$, and for every $f: X \rightarrow \boldsymbol{T} Y$, let $f^{\dagger}\left(F^{-}, F^{+}\right)=\left(\lambda h \in\left\langle Y \rightarrow \mathbb{R}^{+}\right\rangle \cdot F^{-}\left(\lambda x \in X \cdot f^{-}(x)(h)\right), \lambda h \in\langle Y \rightarrow\right.$ $\left.\left.\mathbb{R}^{+}\right\rangle \cdot F^{+}\left(\lambda x \in X \cdot f^{+}(x)(h)\right)\right)$, where by convention $f(x)=\left(f^{-}(x), f^{+}(x)\right)$. Then $(\boldsymbol{T}, \boldsymbol{\eta}, \boldsymbol{\mu})$ is a monad on Top. Together with $\boldsymbol{t}_{X, Y}: X \times \boldsymbol{T} Y \rightarrow \boldsymbol{T}(X \times Y)$ defined by $\boldsymbol{t}_{X, Y}\left(x,\left(F^{-}, F^{+}\right)\right)=\left(\lambda h \in\left\langle Y \rightarrow \mathbb{R}^{+}\right\rangle \cdot F^{-}(\lambda y \in Y \cdot h(x, y)), \lambda h \in\langle Y \rightarrow\right.$ $\left.\left.\mathbb{R}^{+}\right\rangle \cdot F^{+}(\lambda y \in Y \cdot h(x, y))\right)$, it forms a strong monad on Cpo and Pcpo.

Proof. That the strong monad laws are satisfied is obvious: this is just the product of two strong monads as described in Theorem 2. The only thing to check is that unit, extension, and tensorial strength are well defined, i.e., (3) holds for objects meant to be in some space $T Z$.

Unit. Let $F^{-}=F^{+}=\lambda h \in\left\langle X \rightarrow \mathbb{R}^{+}\right\rangle \cdot h(x)=\alpha_{\mathfrak{e}}\left(\delta_{x}\right)$. This is a linear prevision, hence (3) is trivial.

Extension. Recall that $f^{\dagger}\left(F^{-}, F^{+}\right)=\left({F^{\prime}}^{-}, F^{\prime+}\right)$, where ${F^{\prime}}^{-}=\lambda h \in\langle Y \rightarrow$ $\left.\mathbb{R}^{+}\right\rangle \cdot F^{-}\left(\lambda x \in X \cdot f^{-}(x)(h)\right)$ and ${F^{\prime}}^{+}=\lambda h \in\left\langle Y \rightarrow \mathbb{R}^{+}\right\rangle \cdot F^{+}\left(\lambda x \in X \cdot f^{+}(x)(h)\right))$. Then:

$$
\begin{aligned}
F^{\prime-}\left(h+h^{\prime}\right)= & \lambda h \in\left\langle Y \rightarrow \mathbb{R}^{+}\right\rangle \cdot F^{-}\left(\lambda x \in X \cdot f^{-}(x)\left(h+h^{\prime}\right)\right) \\
\leq & \lambda h \in\left\langle Y \rightarrow \mathbb{R}^{+}\right\rangle \cdot F^{-}\left(\lambda x \in X \cdot f^{-}(x)(h)+f^{+}(x)\left(h^{\prime}\right)\right) \\
& \quad \text { since } f(x)=\left(f^{-}(x), f^{+}(x)\right) \in \boldsymbol{T} Y \text { and } F^{-} \text {is monotonic } \\
\leq & \lambda h \in\left\langle Y \rightarrow \mathbb{R}^{+}\right\rangle \cdot F^{-}\left(\lambda x \in X \cdot f^{-}(x)(h)\right)+F^{+}\left(\lambda x \in X \cdot f^{+}(x)\left(h^{\prime}\right)\right) \\
& \quad \text { since }\left(F^{-}, F^{+}\right) \in \boldsymbol{T} X \\
= & F^{\prime-}(h)+F^{\prime+}\left(h^{\prime}\right)
\end{aligned}
$$

We show similarly that ${F^{\prime}}^{-}(h)+{F^{\prime}}^{+}\left(h^{\prime}\right) \leq{F^{\prime}}^{+}\left(h+h^{\prime}\right)$.

Tensorial strength. Recall that $\boldsymbol{t}_{X, Y}\left(x,\left(F^{-}, F^{+}\right)\right)=\left({F^{\prime}}^{-},{F^{\prime}}^{+}\right)$where ${F^{\prime *}}^{*}=$ $\lambda h \in\left\langle Y \rightarrow \mathbb{R}^{+}\right\rangle \cdot F^{*}(\lambda y \in Y \cdot h(x, y))(*$ being - or +$)$. So:

$$
\begin{aligned}
{F^{\prime}}^{-}\left(h+h^{\prime}\right) & =F^{-}\left(\lambda y \in Y \cdot h(x, y)+h^{\prime}(x, y)\right) \\
& \leq F^{-}(\lambda y \in Y \cdot h(x, y))+F^{+}\left(\lambda y \in Y \cdot h^{\prime}(x, y)\right)={F^{\prime}}^{-}(h)+{F^{\prime}}^{+}\left(h^{\prime}\right)
\end{aligned}
$$

since $\left(F^{-}, F^{+}\right) \in \boldsymbol{T}(X \times Y)$; and similarly ${F^{\prime}}^{-}(h)+{F^{\prime}}^{+}\left(h^{\prime}\right) \leq{F^{\prime}}^{+}\left(h+h^{\prime}\right)$. 
Theorem 3. Let $X$ be a stably locally compact space, $F$ a continuous lower prevision, and $f$ a bounded continuous function from $X$ to $\mathbb{R}^{+}$. Then there is a continuous linear functional $G$ from $\left\langle X \rightarrow \mathbb{R}^{+}\right\rangle$to $\overline{\mathbb{R}}^{+}$such that $F \leq G$ and $F(f)=G(f)$.

Proof. Recall that a cone is a set $C$ with two binary operations $+: C \times C \rightarrow C$ and $\cdot: \mathbb{R}^{+} \times C \rightarrow C$, and a constant $0 \in C$ such that $(C,+, 0)$ is a commutative monoid, and $\cdot$ defines an action of $\left(\mathbb{R}^{+}, \times\right)$of $\mathbb{R}^{+}$on $C$ such that additionally $(r+s) \cdot a=$ $r \cdot a+s \cdot a$. An ordered cone if a cone with a partial ordering $\leq$ such that + and $\cdot$ are monotonic in all their arguments. $\mathbb{R}^{+}$, and $\overline{\mathbb{R}}^{+}=\mathbb{R}^{+} \cup\{+\infty\}$ are ordered cones. It is clear that $\left\langle X \rightarrow \mathbb{R}^{+}\right\rangle$is an ordered cone, too. A function $p: C \rightarrow \overline{\mathbb{R}}^{+}$is sub-linear iff $p(r \cdot a)=r p(a)$ for every $r \in \mathbb{R}^{+}$and $p(a+b) \leq p(a)+p(b)$, for all $a, b \in C$. It is super-linear iff $p(a+b) \geq p(a)+p(b)$ instead, and that is is linear if equality holds.

Let $F$ be a lower prevision on $X$, and $f \in\left\langle X \rightarrow \mathbb{R}^{+}\right\rangle$. Let, for every $g \in\langle X \rightarrow$ $\left.\mathbb{R}^{+}\right\rangle$:

$$
\breve{F}_{f}(g)=\inf _{\substack{\lambda \in \mathbb{R}^{+} \\ \lambda f \geq g}}\left[F(\lambda f)-\sup _{\substack{h \in\left\langle X \rightarrow \mathbb{R}^{+}\right\rangle \\ g+h \leq \lambda f}} F(h)\right]
$$

where we take the convention that this is equal to $+\infty$ if there is no $\lambda \in \mathbb{R}^{+}$such that $\lambda f \geq g$.

We shall abbreviate this as $\breve{F}_{f}(g)=\inf _{\lambda / \lambda f \geq g}\left[F(\lambda f)-\sup _{h<\lambda f-g} F(h)\right]$. Note that we cannot in general write $F(\lambda f-g)$ instead of $\sup _{h \leq \lambda f-g} F(h)$, since $\lambda f-g$ is not in general continuous from $X$ to $\mathbb{R}^{+}$(with its Scott topology).

Claim B. $\breve{F}_{f}(0)=0$.

Proof.

$$
\breve{F}_{f}(0)=\inf _{\lambda \in \mathbb{R}^{+}}\left[F(\lambda f)-\sup _{h \leq \lambda f} F(h)\right]=\inf _{\lambda \in \mathbb{R}^{+}}[F(\lambda f)-F(\lambda f)]=0
$$

Claim C. $\breve{F}_{f}$ is monotonic.

Proof. Let $g, g^{\prime} \in\left\langle X \rightarrow \mathbb{R}^{+}\right\rangle$, with $g \leq g^{\prime}$. Fix $\lambda \in \mathbb{R}^{+}$such that $\lambda f \geq g^{\prime}$. For every $h^{\prime} \leq \lambda f-g^{\prime}$ in $\left\langle X \rightarrow \mathbb{R}^{+}\right\rangle$, there is an $h \leq \lambda f-g$ in $\left\langle X \rightarrow \mathbb{R}^{+}\right\rangle$such that $F(h) \geq F\left(h^{\prime}\right)$, namely $h^{\prime}$ itself. So $\sup _{h \leq \lambda f-g} F(h) \geq \sup _{h^{\prime} \leq \lambda f-g^{\prime}} F\left(h^{\prime}\right)$. So $F(\lambda f)-\sup _{h \leq \lambda f-g} F(h) \leq F(\lambda f)-\sup _{h^{\prime} \leq \lambda f-g^{\prime}} F\left(h^{\prime}\right)$. By making $\lambda$ vary,

$$
\inf _{\lambda / \lambda f \geq g^{\prime}}\left[F(\lambda f)-\sup _{h \leq \lambda f-g} F(h)\right] \leq \inf _{\lambda / \lambda f \geq g^{\prime}}\left[F(\lambda f)-\sup _{h^{\prime} \leq \lambda f-g^{\prime}} F\left(h^{\prime}\right)\right]
$$

Since $\lambda f \geq g^{\prime}$ implies $\lambda f \geq g$, the left-hand side of the above inequality is at least:

$$
\inf _{\lambda / \lambda f \geq g}\left[F(\lambda f)-\sup _{h \leq \lambda f-g} F(h)\right]
$$

that is, $\breve{F}_{f}(g)$. The right-hand side is by definition $\breve{F}_{f}\left(g^{\prime}\right)$, so $\breve{F}_{f}(g) \leq \breve{F}_{f}\left(g^{\prime}\right)$. 
Claim D. Let $F$ be a lower prevision on $X . \breve{F}_{f}$ is convex: for every real $\alpha, 0 \leq \alpha \leq$ 1 , for every $g \in\left\langle X \rightarrow \mathbb{R}^{+}\right\rangle$,

$$
\breve{F}_{f}\left(\alpha g+(1-\alpha) g^{\prime}\right) \leq \alpha \breve{F}_{f}(g)+(1-\alpha) \breve{F}_{f}\left(g^{\prime}\right)
$$

Proof. The inequality is clear if $\alpha=0$ or $\alpha=1$. So assume $0<\alpha<1$.

If there is no $\lambda \in \mathbb{R}^{+}$such that $\lambda f \geq g$, or if there is no $\lambda^{\prime} \in \mathbb{R}^{+}$such that $\lambda^{\prime} f \geq g^{\prime}$, the right-hand side of (5) is $+\infty$, so the inequality is vacuously true. So let us assume that for some $\lambda \in \mathbb{R}^{+}, \lambda f \geq g$ and for some $\lambda^{\prime} \in \mathbb{R}^{+}, \lambda^{\prime} f \geq g^{\prime}$.

Let us fix $\lambda$ and $\lambda^{\prime}$ for now. To ease reading, define the following abbreviations: $g^{\prime \prime}=\alpha g+(1-\alpha) g^{\prime}$, and let $\lambda^{\prime \prime}=\alpha \lambda+(1-\alpha) \lambda^{\prime}$.

For every $h \leq \lambda f-g$ in $\left\langle X \rightarrow \mathbb{R}^{+}\right\rangle$, for every $h^{\prime} \leq \lambda^{\prime} f-g^{\prime}$ in $\left\langle X \rightarrow \mathbb{R}^{+}\right\rangle$, let $h^{\prime \prime}=\alpha h+(1-\alpha) h^{\prime}$. The map $h^{\prime \prime}$ is continuous, since $\alpha$ and $1-\alpha$ are nonnegative, multiplication by non-negative reals is Scott-continuous, and addition is also Scott-continuous. Also, $h^{\prime \prime} \leq \lambda^{\prime \prime} f-g^{\prime \prime}$. Finally, since $F$ is a lower prevision, $F$ is concave, i.e., $\alpha F(h)+(1-\alpha) F\left(h^{\prime}\right) \leq F\left(h^{\prime \prime}\right)$. We have just shown that for every $h \leq \lambda f-g$ et $h^{\prime} \leq \lambda^{\prime} f-g^{\prime}$, there is an $h^{\prime \prime} \leq \lambda^{\prime \prime} f-g^{\prime \prime}$ such that $\alpha F(h)+(1-\alpha) F\left(h^{\prime}\right) \leq F\left(h^{\prime \prime}\right)$. It follows:

$$
\sup _{h^{\prime \prime} \leq \lambda^{\prime \prime} f-g^{\prime \prime}} F\left(h^{\prime \prime}\right) \geq \alpha \sup _{h \leq \lambda f-g} F(h)+(1-\alpha) \sup _{h^{\prime} \leq \lambda^{\prime} f-g^{\prime}} F\left(h^{\prime}\right)
$$

Since $\lambda^{\prime \prime}=\alpha \lambda+(1-\alpha) \lambda^{\prime}$,

$$
\begin{aligned}
F\left(\lambda^{\prime \prime} f\right)-\sup _{h^{\prime \prime} \leq \lambda^{\prime \prime} f-g^{\prime \prime}} F\left(h^{\prime \prime}\right) \leq & \alpha\left[F(\lambda f)-\sup _{h \leq \lambda f-g} F(h)\right] \\
& +(1-\alpha)\left[F\left(\lambda^{\prime} f\right)-\sup _{h^{\prime} \leq \lambda^{\prime} f-g^{\prime}} F\left(h^{\prime}\right)\right]
\end{aligned}
$$

By making $\lambda$ and $\lambda^{\prime}$, we obtain:

$$
\begin{aligned}
& \inf _{\substack{\lambda, \lambda^{\prime} \in \mathbb{R}^{+} \\
\lambda f \geq g, \lambda^{\prime} f \geq g^{\prime} \\
\lambda^{\prime \prime}=\alpha \lambda+(1-\alpha) \lambda^{\prime}}}\left[F\left(\lambda^{\prime \prime} f\right)-\sup _{h^{\prime \prime} \leq \lambda^{\prime \prime} f-g^{\prime \prime}} F\left(h^{\prime \prime}\right)\right] \\
\leq & \inf _{\substack{\lambda, \lambda^{\prime} \in \mathbb{R}^{+} \\
\lambda f \geq g, \lambda^{\prime} f \geq g^{\prime}}}\left[\alpha\left[F(\lambda f)-\sup _{h \leq \lambda f-g} F(h)\right]+(1-\alpha)\left[F\left(\lambda^{\prime} f\right)-\sup _{h^{\prime} \leq \lambda^{\prime} f-g^{\prime}} F\left(h^{\prime}\right)\right]\right]
\end{aligned}
$$

Clearly $\lambda^{\prime \prime} \in \mathbb{R}^{+}$and $\lambda^{\prime \prime} f \geq g^{\prime \prime}$. Recall that there exist $\lambda \in \mathbb{R}^{+}$such that $\lambda f \geq g$ and $\lambda^{\prime} \in \mathbb{R}^{+}$such that $\lambda^{\prime} f \geq g^{\prime}$. The right hand side of (6) therefore equals $\alpha \breve{F}_{f}(g)+(1-\alpha) \breve{F}_{f}\left(g^{\prime}\right)$. For the left hand side, observe that for every $\lambda, \lambda^{\prime} \in \mathbb{R}^{+}$ such that $\lambda f \geq g, \lambda^{\prime} f \geq g^{\prime}$, the quantity $\lambda^{\prime \prime}=\alpha \lambda+(1-\alpha) \lambda^{\prime}$ is such that $\lambda^{\prime \prime} \in \mathbb{R}^{+}$and $\lambda^{\prime \prime} f \geq g^{\prime \prime}$ : the left hand side of (6) is in particular greater than or equal to the greatest lower bound, over all $\lambda^{\prime \prime} \in \mathbb{R}^{+}$such that $\lambda^{\prime \prime} f \geq g^{\prime \prime}$, of $F\left(\lambda^{\prime \prime} f\right)-\sup _{h^{\prime \prime} \leq \lambda^{\prime \prime} f-g^{\prime \prime}} F\left(h^{\prime \prime}\right)$. But this greatest lower bound is exactly $\widetilde{F}_{f}\left(g^{\prime \prime}\right)$. So $\breve{F}_{f}\left(g^{\prime \prime}\right) \leq \alpha \breve{F}_{f}(g)+(1-\alpha) \breve{F}_{f}\left(g^{\prime}\right)$. 
Claim E. Let $F$ be a lower prevision on $X . \breve{F}_{f}$ is positively homogeneous: for every $\alpha \geq 0, \breve{F}_{f}(\alpha g)=\alpha \breve{F}_{f}(g)$.

Proof. When $\alpha=0$, this is by Claim B. For all $\alpha>0$,

$$
\begin{aligned}
\breve{F}_{f}(\alpha g) & =\inf _{\lambda \in \mathbb{R}^{+}, \lambda f \geq \alpha g}\left[F(\lambda f)-\sup _{h \leq \lambda f-\alpha g} F(h)\right] \\
& =\inf _{\lambda^{\prime} \in \mathbb{R}^{+}, \lambda^{\prime} f \geq g}\left[F\left(\alpha \lambda^{\prime} f\right)-\sup _{h \leq \alpha \lambda^{\prime} f-\alpha g} F(h)\right] \quad\left(\text { where } \lambda^{\prime}=\lambda / \alpha\right) \\
& =\inf _{\lambda^{\prime} \in \mathbb{R}^{+}, \lambda^{\prime} f \geq g}\left[F\left(\alpha \lambda^{\prime} f\right)-\sup _{h^{\prime} \leq \lambda^{\prime} f-g} F\left(\alpha h^{\prime}\right)\right] \quad\left(\text { where } h^{\prime}=h / \alpha\right) \\
& =\inf _{\lambda^{\prime} \in \mathbb{R}^{+}, \lambda^{\prime} f \geq g}\left[\alpha F\left(\lambda^{\prime} f\right)-\sup _{h^{\prime} \leq \lambda^{\prime} f-g} \alpha F\left(h^{\prime}\right)\right]
\end{aligned}
$$

since $F$ is positively homogeneous. But this is exactly $\alpha \breve{F}_{f}(g)$.

Claim F. Let $F$ be a lower prevision on $X$. For every $g \in\left\langle X \rightarrow \mathbb{R}^{+}\right\rangle, \breve{F}_{f}(g) \geq$ $F(g)$.

Proof. For every $\lambda \in \mathbb{R}^{+}$such that $\lambda f \geq g$, for every $h \leq \lambda f-g, F(\lambda f) \geq$ $F(h)+F(g)$. Indeed, $F(h)+F(g) \leq F(h+g)$ since $F$ is super-additive, and $F(h+g) \leq F(\lambda f)$ by assumption.

So $F(\lambda f) \geq \sup _{h \leq \lambda f-g} F(h)+F(g)$, i.e., $F(\lambda f)-\sup _{h \leq \lambda f-g} F(h) \geq F(g)$. We conclude by taking greatest lower bounds over all the $\lambda \in \mathbb{R}^{+}$such that $\lambda f \geq g$.

Claim G. Let $F$ be a lower prevision on $X$. Then $\breve{F}_{f}(f)=F(f)$.

Proof. If $f$ is the 0 function, then $\breve{F}_{f}(f)=F(f)=0$. Otherwise, the smallest $\lambda \in$ $\mathbb{R}^{+}$such that $\lambda f \geq f$ is 1 , so $\breve{F}_{f}(f)=\inf _{\lambda / \lambda f \geq f}\left[F(\lambda f)-\sup _{h \leq \lambda f-f} F(h)\right]=$ $F(1 . f)-F(0)=F(f)$.

Although $\breve{F}_{f}$ may take have $+\infty$ as value, it is not the case if $f$ is bounded away from 0 from below:

Claim H. Let $F$ be a lower prevision on $X . \breve{F}_{f}\left(\chi_{X}\right) \geq F\left(\chi_{X}\right)$. Moreover, if $\inf _{x \in X} f(x)>0$, then $\breve{F}_{f}\left(\chi_{X}\right) \leq \frac{1}{\inf _{x \in X} f(x)} F(f)$.

Proof.

$$
\begin{aligned}
\breve{F}_{f}\left(\chi_{X}\right) & =\inf _{\lambda / \lambda f \geq \chi_{X}}\left[F(\lambda f)-\sup _{h \leq \lambda f-\chi_{X}} F(h)\right] \\
& =\inf _{\lambda / \lambda f \geq \chi_{X}}\left[F(\lambda f)-F\left(\lambda f-\chi_{X}\right)\right]
\end{aligned}
$$

We observe indeed that $h=\lambda f-\chi_{X}=\lambda f-1$ is continuous. Since $F$ is concave, $F(\lambda f) \geq F\left(\lambda f-\chi_{X}\right)+F\left(\chi_{X}\right)$, so $\breve{F}_{f}\left(\chi_{X}\right) \geq F\left(\chi_{X}\right)$. 
As far as the second inequality is concerned, since $F\left(\lambda f-\chi_{X}\right) \geq 0$, we obtain $\breve{F}_{f}\left(\chi_{X}\right) \leq \inf _{\lambda / \lambda f \geq \chi_{X}} F(\lambda f)$. Let $a_{0}=\inf _{x \in X} f(x)$, and note that $\lambda f \geq \chi_{X}$ iff $\lambda \geq 1 / a_{0}$. So $\breve{F}_{f}\left(\chi_{X}\right) \leq 1 / a_{0} F(f)$.

We can then use Roth's Sandwich Theorem to conclude that there is a linear $G$ such that $F \leq G$ and satisfying some added conditions. For now, $G$ may take the value $+\infty$, and is not necessarily continuous. In a picturesque way, such a $G$ will be called an upper tangent.

Claim I. Let $F$ be a lower prevision on $X$, and $f$ a bounded continuous function from $X$ to $\mathbb{R}^{+}$. There is a functional $G$ from $\left\langle X \rightarrow \mathbb{R}^{+}\right\rangle$to $\overline{\mathbb{R}}^{+}$, which is linear and monotonic, and such that $F \leq G$ and $F(f)=G(f)$. Furthermore, if $\inf _{x \in X} f(x) \neq 0$, then $G$ is a linear prevision.

Proof. By Claim E and Claim D, $\breve{F}_{f}$ is sub-linear. By assumption, $F$ is super-linear. Moreover, $F$ and $\breve{F}_{f}$ are monotonic and $F \leq \breve{F}_{f}$ by Claim F. By Roth's Sandwich Theorem, there is a functional $G$ from $\left\langle X \rightarrow \mathbb{R}^{+}\right\rangle$to $\overline{\mathbb{R}}^{+}$, which is monotonic and linear, and such that $F \leq G \leq \breve{F}_{f}$. By Claim $\mathrm{G}, F(f) \leq G(f) \leq \breve{F}_{f}(f)=F(f)$, so $G(f)=F(f)$.

If moreover inf $\operatorname{meX}_{x \in X} f(x) \neq 0$, by Claim $\mathrm{H}, G\left(\chi_{X}\right) \leq \breve{F}_{f}\left(\chi_{X}\right) \leq 1 / \inf _{x \in X} f(x) F(f)<$ $+\infty$. So for every $g \in\left\langle X \rightarrow \mathbb{R}^{+}\right\rangle, G(g) \leq G\left(\sup _{x \in X} g(x) \chi_{X}\right)<+\infty$. Since $G$ takes its values in $\mathbb{R}^{+}$, no longer in $\overline{\mathbb{R}}^{+}, G$ is a linear prevision.

To attack continuous previsions, we first need to explore the structure of the space $\left\langle X \rightarrow \mathbb{R}^{+}\right\rangle$. This is probably well-known. In doubt, I preferred to produce proofs. Let $\Subset$ be the way-below relation on the set $\mathcal{O}(X)$ of opens of $X$, ordered by inclusion. $X$ is a core compact space iff $\mathcal{O}(X)$ is a continuous cpo [2]. This is in particular the case when $X$ is locally compact, where $U \Subset V$ iff $U \subseteq Q \subseteq V$ for some saturated compact subset $Q$ of $X$.

Claim J. Let $\ll$ be the way-below relation of $\left\langle X \rightarrow \mathbb{R}^{+}\right\rangle$, and $\ll_{1}$ that of $\langle X \rightarrow$ $[0,1]\rangle$ of all continuous functions $f$ bounded from above by 1 .

Let $f=\sum_{i=1}^{n} a_{i} \chi_{U_{i}}$ a step function from $X$ to $\mathbb{R}^{+}$(resp. $\left.[0,1]\right), U_{1} \supseteq \ldots \supseteq U_{n}$, $a_{1}, \ldots, a_{n} \in \mathbb{R}^{+} \backslash\{0\}$. Let $g$ a continuous function from $X$ to $\mathbb{R}^{+}$(resp. $[0,1]$ ).

Then $f \ll g$ (resp. $f \ll 1 g$ ) iff for every $i, 1 \leq i \leq n, U_{i} \Subset g^{-1}\left(\sum_{j=1}^{i} a_{i},+\infty\right)$.

Proof. Step 1. The condition is necessary. Let us indeed fix $i, 1 \leq i \leq n$, let $t_{i}$ be

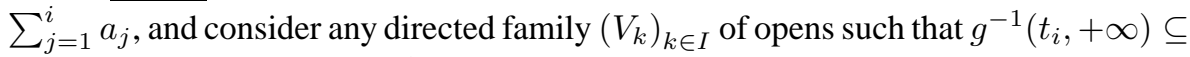
$\bigcup_{k \in I} V_{k}$. Let $W_{k}=V_{k} \cap g^{-1}\left(t_{i},+\infty\right)$. For each $k \in I$ and each real $r, 0<r<1$, let $f_{r, k}$ be the function $r .\left(\max \left(\min \left(t_{i}, g\right), \chi_{W_{k}} . g\right)\right)$. This is continuous, as a composition of continuous functions. Note in particular that max, min and multiplication by a non-negative scalar are Scott-continuous. The only thing to check really is that $\chi_{W_{k}} . g$ is continuous: the inverse image of $(t,+\infty)$ is $X$ if $t<0$, $W_{k} \cap g^{-1}(t,+\infty)$ otherwise, which is indeed open. Note also that, in case we consider functions in $\langle X \rightarrow[0,1]\rangle$, and $f \ll_{1} g$, then $f_{r, k}$ is again in $\langle X \rightarrow[0,1]\rangle$. If $x \in W_{k} \subseteq g^{-1}\left(t_{i},+\infty\right), f_{r, k}(x)=r . g(x)$; if $x \in g^{-1}\left(t_{i},+\infty\right) \backslash W_{k}, f_{r, k}(x)=$ $r . t_{i}$; if $x \notin g^{-1}\left(t_{i},+\infty\right)$ (in particular $\left.x \notin W_{k}\right), f_{r, k}(x)=r . g(x)$. 
Note that if $r \leq r^{\prime}$ and $V_{k} \subseteq V_{k^{\prime}}$ (so $W_{k} \subseteq W_{k^{\prime}}$ ), then $f_{r, k} \leq f_{r^{\prime}, k^{\prime}}$ : if $x \in W_{k}$ then $f_{r, k}(x)=r . g(x) \leq r^{\prime} . g(x)=f_{r^{\prime}, k^{\prime}}(x)$, since $x \in W_{k^{\prime}}$; if $x \in W_{k^{\prime}} \backslash W_{k}$, then $\left.x \in g^{-1}\right] t_{i},+\infty\left[\backslash W_{k}\right.$, so $f_{r, k}(x)=r . t_{i}<r^{\prime} . g(x)=f_{r^{\prime}, k^{\prime}}(x)$; if $x \in$ $g^{-1}\left(t_{i},+\infty\right) \backslash W_{k^{\prime}}, f_{r, k}(x)=r . t_{i} \leq r^{\prime} . t_{i}=f_{r^{\prime}, k^{\prime}}(x)$; and is $x \notin g^{-1}\left(t_{i},+\infty\right)$, then $f_{r, k}(x)=r . g(x) \leq r^{\prime} . g(x)=f_{r^{\prime}, k^{\prime}}(x)$. It follows that the family $\left(f_{r, k}\right)_{\substack{0<r<1 \\ k \in I}}$ is directed, since $f_{r, k}$ and $f_{r^{\prime}, k^{\prime}}$ are both less than or equal to $f_{\max \left(r, r^{\prime}\right), k^{\prime \prime}}$, where $k^{\prime \prime}$ is such that $V_{k}, V_{k^{\prime}} \subseteq V_{k^{\prime \prime}}$.

Moreover, the least upper bound of this family is exactly $g$ : for every $x \in X$, either $x \in g^{-1}\left(t_{i},+\infty\right)=\bigcup_{k \in I} W_{k}$, so there is $k \in I$ with $x \in W_{k}$, and then $f_{r, k}(x)=r . g(x)$; or $x \notin g^{-1}\left(t_{i},+\infty\right)$ and then $f_{r, k}(x)=r . g(x)$ again; but then $\sup _{0<r<1} r . g(x)=g(x)$.

If $f \ll g$, necessarily $f \leq f_{r, k}$ for some $r, 0<r<1$, and some $k \in I$. Then, for every $x \in U_{i}, f(x) \geq t_{i}$, so $f_{r, k}(x) \geq t_{i}$. By definition of $f_{r, k}$, this entails $\max \left(\min \left(t_{i}, g(x)\right), \chi_{W_{k}}(x) . g(x)\right) \geq t_{i} / r$. Since $\min \left(t_{i}, g(x)\right) \leq t_{i}<t_{i} / r$, necessarily $\chi_{W_{k}}(x) . g(x) \geq t_{i} / r$, so $x \in W_{k}$ and $g(x) \geq t_{i} / r$.

In particular, $U_{i} \subseteq W_{k} \subseteq V_{k}$. Since the family $\left(V_{k}\right)_{k \in I}$ is arbitrary, $U_{i} \Subset g^{-1}\left(t_{i},+\infty\right)$. Step 2. Conversely, the condition is sufficient. Indeed, assume that for every $i, 1 \leq$ $\overline{i \leq n,} U_{i} \Subset g^{-1}\left(t_{i},+\infty\right)$, where, as above, $t_{i}=\sum_{j=1}^{i-1} a_{i}$.

Let us show that $f \ll g$ (resp. $f \ll_{1} g$ ). Let $\left(f_{k}\right)_{k \in I}$ a directed family of continuous functions from $X$ to $\mathbb{R}^{+}$(resp. [0,1]) such that $g \leq \sup _{k \in I} f_{k}$.

For every $t \in \mathbb{R}^{+}, g^{-1}(t,+\infty)=\{x \in X \mid g(x)>t\} \subseteq\left\{x \in X \mid \sup _{k \in I} f_{k}(x)>\right.$ $t\}=\left\{x \in X \mid \exists k \in I \cdot f_{k}(x)>t\right\}=\bigcup_{k \in I} f_{k}^{-1}(t,+\infty)$. The family $\left(f_{k}^{-1}(t,+\infty)\right)_{k \in I}$ is directed, since $\left(f_{k}\right)_{k \in I}$ is directed and $f_{k} \leq f_{k^{\prime}}$ implies $f_{k}^{-1}(t,+\infty) \subseteq f_{k^{\prime}}^{-1}(t,+\infty)$. Since $U_{i} \Subset g^{-1}\left(t_{i},+\infty\right) \subseteq \bigcup_{k \in I} f_{k}^{-1}\left(t_{i},+\infty\right)$, there is a $k_{i} \in I$ such that $U_{i} \subseteq f_{k_{i}}^{-1}\left(t_{i},+\infty\right)$. Since $\left(f_{k}\right)_{k \in I}$ is directed, there is a $k \in I$ such that $f_{k_{i}} \leq f_{k}$ for every $i, 1 \leq i \leq n$. So $U_{i} \subseteq f_{k_{i}}^{-1}\left(t_{i},+\infty\right) \subseteq f_{k}^{-1}\left(t_{i},+\infty\right)$.

Let $U_{0}=X, U_{n+1}=\emptyset$. For every $x \in X$, let $i$ be the unique natural number between 0 and $n$ such that $x \in U_{i} \backslash U_{i+1}$. If $i=0$, then $f(x)=0$, so $f(x) \leq f_{k}(x)$. If $i \neq 0$, then $f(x)=\sum_{j=1}^{i} a_{i}=t_{i}$, and since $x \in U_{i}, x \in f_{k}^{-1}\left(t_{i},+\infty\right)$, i.e., $f_{k}(x)>t_{i}$. In other words, $f_{k}(x)>f(x)$. Since $x$ is arbitrary, $f_{k} \geq f$. So $f \ll g$.

Recall that a basis $B$ of a continuous poset $Y$ is any subset of $Y$ such that every element of $x$ can be written as a directed least upper bound of elements in $B$.

Claim K. Let $X$ be a core compact space. Let $B$ the set of step functions of the form $1 / 2^{K} \sum_{k=1}^{N} \chi_{U_{k}}, K, N \in \mathbb{N}$, where $\left(U_{k}\right)_{k=1}^{2^{K}}$ is a decreasing sequence of opens of $X$.

Then $\left\langle X \rightarrow \mathbb{R}^{+}\right\rangle$is a continuous poset, with basis $B$. The space $\langle X \rightarrow[0,1]\rangle$ is a continuous cpo, with basis $B_{1}=B \cap\langle X \rightarrow[0,1]\rangle$.

Proof. Let $f \in\left\langle X \rightarrow \mathbb{R}^{+}\right\rangle$, resp. $f \in\langle X \rightarrow[0,1]\rangle$. Consider the set $D$ of all functions of the form $1 / 2^{K} \sum_{k=1}^{N} \chi_{U_{k}}, K, N \in \mathbb{N}$, where $U_{k} \Subset f^{-1}\left(k / 2^{K},+\infty\right)$ for every $k, 1 \leq k \leq N$. Clearly $D \subseteq B$, resp. $D \subseteq B_{1}$.

We first show that $D$ is directed. $D$ is non-empty: take $N=0$. Then, if $1 / 2^{K} \sum_{k=1}^{N} \chi_{U_{k}}$ and $1 / 2^{K^{\prime}} \sum_{k=1}^{N^{\prime}} \chi_{V_{k}}$ are in $D$, we may assume wlog. that $K=K^{\prime}$ and $N=N^{\prime}$. 
Let us show that we may require $K=K^{\prime}$ : if, say, $K^{\prime}<K$, one can rewrite the second function as $1 / 2^{K} \sum_{k=1}^{2^{K-K^{\prime}} N^{\prime}} \chi_{W_{k}}$, where $W_{k}=V_{\left\lfloor\left(k+2^{K-K^{\prime}}-1\right) / 2^{K-K^{\prime}}\right\rfloor}$ for every $k, 1 \leq k \leq 2^{K-K^{\prime}} N^{\prime}$. It follows from $K=K^{\prime}$ that we may also require $N=N^{\prime}$ : if $N^{\prime}<N$, for example, then $1 / 2^{K^{\prime}} \sum_{k=1}^{N^{\prime}} \chi_{V_{k}}=1 / 2^{K^{\prime}} \sum_{k=1}^{N} \chi_{V_{k}}$, where we let $V_{k}=\emptyset$ for each $k, N^{\prime}<k \leq N$.

Given any two functions of the form $1 / 2^{K} \sum_{k=1}^{N} \chi_{U_{k}}$ and $1 / 2^{K} \sum_{k=1}^{N} \chi_{V_{k}}$ in $D$, then $1 / 2^{K} \sum_{k=1}^{N} \chi_{U_{k} \cup V_{k}}$ is again in $D$, since $U_{k} \cup V_{k} \Subset f^{-1}\left(k / 2^{K},+\infty\right)$. (In any core compact space, $U \cup V \Subset W$ as soon as $U \Subset W$ and $V \Subset W$ [2].) So $D$ is directed.

Now $f$ is the least upper bound of the sequence $f_{K}=1 / 2^{K} \sum_{k=1}^{N} \chi_{\left.f^{-1}\right] k / 2^{K},+\infty[}$, $K \in \mathbb{N}, N=\left\lfloor a 2^{K}\right\rfloor$, where $a=\sup _{x \in X} f(x)$. Since $\mathcal{O}(X)$ is a continuous cpo, $f^{-1}\left(k / 2^{K},+\infty\right)$ is the directed union of all opens $U \Subset f^{-1}\left(k / 2^{K},+\infty\right)$, so $\chi_{f^{-1}\left(k / 2^{K},+\infty\right)}=\sup _{U \Subset f^{-1}\left(k / 2^{K},+\infty\right)} \chi_{U}$. So $f_{K}$ is a least upper bound of elements in $D$. Since $f=\sup _{K \in \mathbb{N}} f_{K}, f$ is also a directed least upper bound of elements in $D$. By Claim $\mathrm{J}$, every element in $D$ is way-below $f$.

In particular, whenever $f \ll g$ then there is a step function $f^{\prime} \in B$ such that $f \ll f^{\prime} \ll g$. This is the interpolation property, valid in any continuous poset [15].

The following states that $\ll$ is multiplicative:

Claim L. Let $f$ be any step function from $X$ to $\mathbb{R}^{+}, g$ a continuous function from $X$ to $\mathbb{R}^{+}$, and $a>0$ a real such that $a \geq \sup _{x \in X} f(x), a \geq \sup _{x \in X} g(x)$. Then $f \ll g$ iff $f / a \ll_{1} g / a$.

Proof. Let $f=\sum_{i=1}^{n} a_{i} \chi_{U_{i}}$. Then $f \ll g$ iff for every $i, 1 \leq i \leq n, U_{i} \Subset$ $g^{-1}\left(\sum_{j=1}^{i} a_{i},+\infty\right)$ by Claim J. Again by Claim J, $f / a \ll_{1} g / a$ iff for every $i, 1 \leq$ $i \leq n, U_{i} \Subset(g / a)^{-1}\left(\sum_{j=1}^{i} a_{i} / a,+\infty\right)$. Now note that $(g / a)^{-1}\left(\sum_{j=1}^{i} a_{i} / a,+\infty\right)=$ $g^{-1}\left(\sum_{j=1}^{i} a_{i},+\infty\right)$. So $f \ll g$ is equivalent to $f / a \ll_{1} g / a$.

Let us say that $X$ is core coherent iff for all opens $U, V_{1}, V_{2}$ of $X$, if $U \Subset V_{1}$ and $U \Subset V_{2}$ then $U \Subset V_{1} \cap V_{2}$. We call $X$ stably core compact iff it is both core compact and core coherent. Every stably locally compact space $X$ is stably core compact, and the converse holds if $X$ is sober.

The following states that $\ll$ is additive, provided $X$ is stably core compact.

Claim M. Let $X$ be core compact, and $f, g$ two bounded continuous functions from $X$ to $\mathbb{R}^{+}$. If $h \ll f+g$, then for some $f^{\prime}, g^{\prime} \in B$ we have $h \leq f^{\prime}+g^{\prime}, f^{\prime} \ll f$ and $g^{\prime} \ll g$.

Proof. Let $B_{f}$ the set of all functions $f^{\prime} \in B$ such that $f^{\prime} \leq f, B_{g}$ that of all functions $g^{\prime} \in B$ such that $g^{\prime} \leq g$. By Claim K, $f=\sup _{f^{\prime} \in B_{f}} f^{\prime}, g=\sup _{g^{\prime} \in B_{g}} g^{\prime}$. Since addition is Scott-continuous, $f+g=\sup _{f^{\prime} \in B_{f}, g^{\prime} \in B_{g}} f^{\prime}+g^{\prime}$. Moreover, $B_{f}$ and $B_{g}$ are directed, so $B_{f} \times B_{g}$ as well. Since $h \ll f+g$, there are $f^{\prime} \in B_{f}$ et $g^{\prime} \in B_{g}$ such that $h \leq f^{\prime}+g^{\prime}$.

We now need an explicit formula for sums of functions in $B$.

Claim N. Let $f^{\prime}=1 / 2^{K} \sum_{i=1}^{N} \chi_{A_{i}}, g^{\prime}=1 / 2^{K} \sum_{j=1}^{N^{\prime}} \chi_{B_{j}}$ two functions from $X$ to $\mathbb{R}$, with $X \supseteq A_{1} \supseteq A_{2} \supseteq \ldots \supseteq A_{N}$ and $X \supseteq B_{1} \supseteq B_{2} \supseteq \ldots \supseteq B_{N^{\prime}}$. By 
extension, let $A_{0}=B_{0}=X$, and $A_{i}=\emptyset$ for every $i>N, B_{j}=\emptyset$ for every $j>N^{\prime}$. Then $f^{\prime}+g^{\prime}=1 / 2^{K} \sum_{k=1}^{N+N^{\prime}} \chi_{W_{k}}$, where $W_{k}=\bigcup_{\substack{i \in \mathbb{N}, j \in \mathbb{N} \\ i+j=k}}\left(A_{i} \cap B_{j}\right)$.

Proof. As a side remark, note that choosing the same $K$ for $f^{\prime}$ and $g^{\prime}$ incurs no loss in generality. For every $k \geq 1, f^{\prime}(x)+g^{\prime}(x) \geq k / 2^{K}$ iff there are two indices $i, j$ such that $i+j=k, f^{\prime}(x) \geq i / 2^{K}$, and $g^{\prime}(x) \geq j / 2^{K}$, i.e., such that $i+j=k$, and $x \in A_{i} \cap B_{j}$. So $f^{\prime}+g^{\prime}=1 / 2^{K} \sum_{k>1} \chi_{W_{k}}$. But, for $k>N+N^{\prime}$, whatever $i$ and $j$ such that $i+j=k$, either $i>N$ or $j>N^{\prime}$, so either $U_{i}=\emptyset$ or $V_{j}=\emptyset$, i.e. $W_{k}=\emptyset$. The sum therefore indeed stop at $k=N+N^{\prime}$ at the latest.

Claim O. Let $X$ be a core coherent space. For every bounded continuous functions $f, g$ from $X$ to $\mathbb{R}^{+}$, and every functions $f^{\prime}, g^{\prime}$ in $B$, if $f^{\prime} \ll f$ and $g^{\prime} \ll g$, then $f^{\prime}+g^{\prime} \ll f+g$.

Proof. Write $f^{\prime}=1 / 2^{K} \sum_{i=1}^{N} \chi_{U_{i}}, g^{\prime}=1 / 2^{K} \sum_{j=1}^{N} \chi_{V_{j}}$. As in Claim K, we may assume that $f^{\prime}$ and $g^{\prime}$ are written with the same $K$ and the same $N$. By extension, let $U_{0}=V_{0}=X$, and $U_{i}=V_{i}=\emptyset$ for every $i>N$. By Claim N, $f^{\prime}+g^{\prime}=$ $1 / 2^{K} \sum_{k=1}^{2 N} \chi_{W_{k}}$, where $W_{k}=\bigcup_{i+j=k}\left(U_{i} \cap V_{j}\right)$.

By assumption, $f^{\prime} \ll f$, so by Claim J, $U_{i} \Subset f^{-1}\left(i / 2^{K},+\infty\right)$ for every $i, 1 \leq i \leq$ $2^{K}$. This again holds when $i>2^{K}$, since then $U_{i}$ is empty, therefore way-below any open. Similarly, since $g^{\prime} \ll g, V_{j} \Subset g^{-1}\left(j / 2^{K},+\infty\right)$ pour tout $j \geq 1$.

It follows that $U_{i} \cap V_{j} \Subset(f+g)^{-1}\left(k / 2^{K},+\infty\right)$ for every $i, j \geq 1$ such that $i+j=k$. Indeed, $U_{i} \cap V_{j} \subseteq U_{i} \Subset f^{-1}\left(i / 2^{K},+\infty\right)$ and $U_{i} \cap V_{j} \subseteq V_{j} \Subset$ $g^{-1}\left(j / 2^{K},+\infty\right)$, so $U_{i} \cap V_{j} \Subset f^{-1}\left(i / 2^{K},+\infty\right) \cap g^{-1}\left(j / 2^{K},+\infty\right)$, since $X$ is core coherent. But, for every $x \in f^{-1}\left(i / 2^{K},+\infty\right) \cap g^{-1}\left(j / 2^{K},+\infty\right), f(x)>i / 2^{K}$ and $g(x)>j / 2^{K}$, so $f(x)+g(x)>k / 2^{K}$, i.e., $x \in(f+g)^{-1}\left(k / 2^{K},+\infty\right)$. So $U_{i} \cap V_{j} \Subset f^{-1}\left(i / 2^{K},+\infty\right) \cap g^{-1}\left(j / 2^{K},+\infty\right) \subseteq(f+g)^{-1}\left(k / 2^{K},+\infty\right)$.

We also have $U_{i} \cap V_{j} \Subset(f+g)^{-1}\left(k / 2^{K},+\infty\right)$ when $i+j=k \geq 1$ but $i$ or $j$ is zero. If for example $i=0$, then $j=k, U_{i} \cap V_{j}=X \cap V_{k}=V_{k} \Subset$ $g^{-1}\left(k / 2^{K},+\infty\right) \subseteq(f+g)^{-1}\left(k / 2^{K},+\infty\right)$.

So $U_{i} \cap V_{j} \Subset(f+g)^{-1}\left(k / 2^{K},+\infty\right)$ for every $i, j$ such that $i+j=k, k \geq 1$. It follows easily that $W_{k}=\bigcup_{i+j=k} U_{i} \cap V_{j} \Subset(f+g)^{-1}\left(k / 2^{K},+\infty\right)$ for every $k \geq 1$. By Claim $\mathrm{J}, f^{\prime}+g^{\prime} \ll f+g$.

Claim P. Let $X$ be compact. For every $h^{\prime} \in B$, for every $a>0, h^{\prime} \ll a \chi_{X}$ iff $h^{\prime}$ is of the form $\sum_{i=1}^{n} a_{i} \chi_{U_{i}}, U_{1} \supseteq \ldots \supseteq U_{n}, a_{1}, \ldots, a_{n} \in \mathbb{R}^{+}$, with $\sum_{i=1}^{n} a_{i}<a$. In particular, $h^{\prime} \ll a \chi_{X}$ iff there is $a^{\prime}<a$ with $h^{\prime} \leq a^{\prime} \chi_{X}$.

Proof. For every $h^{\prime} \in B$, write $h^{\prime}=\sum_{i=1}^{n} a_{i} \chi_{U_{i}}, U_{1} \supseteq \ldots \supseteq U_{n}, a_{1}, \ldots, a_{n} \in$ $\mathbb{R}^{+} \backslash\{0\}$. By Claim $\mathrm{J}, h^{\prime} \ll a \chi_{X}$ iff $U_{i} \Subset \emptyset$ for every $i$ such that $\sum_{j=1}^{i} a_{i} \geq a$, and $U_{i} \Subset X$ for every $i$ such that $\sum_{j=1}^{i} a_{i}<a$. This amounts to requiring that $U_{i}=\emptyset$ for every $i$ such that $\sum_{j=1}^{i} a_{i} \geq a$; since $X$ is compact, $U_{i} \Subset X$. The functions $h^{\prime}$ such that $h^{\prime} \ll a \chi_{X}$ are therefore exactly those of the form $\sum_{i=1}^{n} a_{i} \chi_{U_{i}}, U_{1} \supseteq$ $\ldots \supseteq U_{n}$ with $\sum_{i=1}^{n} a_{i}<a$.

If $h^{\prime} \ll a \chi_{X}$, we have $h^{\prime} \leq a^{\prime} \chi_{X}$, where $a^{\prime}$ is any real strictly between $\sum_{i=1}^{n} a_{i}$ and $a$. Conversely, if $h^{\prime} \leq a^{\prime} \chi_{X}$ with $a^{\prime}<a$, then $h^{\prime} \ll a \chi_{X}$ since $a^{\prime} \chi_{X} \ll a \chi_{X}$ by Claim J. 
We now have enough material to apply Scott's formula on the continuous poset $\langle X \rightarrow$ $\left.\mathbb{R}^{+}\right\rangle$, with basis $B$. For any functional $F$ from $\left\langle X \rightarrow \mathbb{R}^{+}\right\rangle$to $\overline{\mathbb{R}}^{+}$, let $\mathfrak{r}(F)$ be the function defined by:

$$
\mathfrak{r}(F)(f)=\sup _{g \in B, g \ll f} F(g)
$$

Then $\mathfrak{r}(F)$ is a continuous functional from $\left\langle X \rightarrow \mathbb{R}^{+}\right\rangle$to $\overline{\mathbb{R}}^{+}$. In fact, $\mathfrak{r}(F)$ is the greatest continuous functional below $F$. We observe:

Claim Q. Let $X$ be stably core compact. If $F$ is a (plain, lower, upper, linear) prevision then $\mathfrak{r}(F)$, too. If moreover $X$ is compact and $F$ is normalized, then $\mathfrak{r}(F)$ is normalized.

Proof. The function $\mathfrak{r}(F)$ takes its values in $\mathbb{R}^{+}$, since if $a=\sup _{x \in X} f(x)$, then $\mathfrak{r}(F)(f)=\sup _{g \in B, g \ll f} F(g) \leq F\left(a \chi_{X}\right)<+\infty$. It is continuous, and is positively homogeneous by Claim L. It is lower as soon as $F$ is, since:

$$
\begin{aligned}
\mathfrak{r}(F)(f+g) & =\sup _{h \in B, h \ll f+g} F(h) \\
& =\sup _{h^{\prime}, h^{\prime \prime} \in B, h^{\prime} \ll f, h^{\prime \prime} \ll g} F\left(h^{\prime}+h^{\prime \prime}\right) \quad \text { by Claim M and Claim O } \\
& \geq \sup _{h^{\prime}, h^{\prime \prime} \in B, h^{\prime} \ll f, h^{\prime \prime} \ll g}\left(F\left(h^{\prime}\right)+F\left(h^{\prime \prime}\right)\right) \\
& =\sup _{h^{\prime} \in B, h^{\prime} \ll f} F\left(h^{\prime}\right)+\sup _{h^{\prime \prime} \in B, h^{\prime \prime} \ll g} F\left(h^{\prime \prime}\right)=\mathfrak{r}(F)(f)+\mathfrak{r}(F)(g)
\end{aligned}
$$

Similarly, if $F$ is upper then $\mathfrak{r}(F)$ is upper too, and if $F$ is linear then so is $\mathfrak{r}(F)$. Finally, if $F$ is normalized and $X$ is compact, then for every $a>0$,

$$
\begin{aligned}
\mathfrak{r}(F)\left(a \chi_{X}+f\right) & =\sup _{h \in B, h \ll a \chi_{X}+f} F(h) \\
& =\sup _{h^{\prime}, h^{\prime \prime} \in B, h^{\prime} \ll a \chi_{X}, h^{\prime \prime} \ll f} F\left(h^{\prime}+h^{\prime \prime}\right) \\
& =\sup _{a^{\prime}<a, h^{\prime \prime} \in B, h^{\prime \prime} \ll f} F\left(a^{\prime} \chi_{X}+h^{\prime \prime}\right) \quad \text { (by Claim P) } \\
& =\sup _{a^{\prime}<a, h^{\prime \prime} \in B, h^{\prime \prime} \ll f}\left(a^{\prime}+F\left(h^{\prime \prime}\right)\right) \quad \text { (since } F \text { is normalized) } \\
& =a+\mathfrak{r}(F)(f)
\end{aligned}
$$

By Claim I, there is a monotonic linear functional $G_{0}$ such that $F \leq G_{0}$ and $F(f)=$ $G_{0}(f)$. Let $G=\mathfrak{r}\left(G_{0}\right)$. By Claim Q, $G$ is a continuous linear functional. Since it is the greatest one below $G_{0} F \leq G$. Finally, $G \leq G_{0}$, so $G(f) \leq F(f)$; since $F \leq G$, $F(f) \leq G(f)$, so $F(f)=G(f)$.

Theorem 4. Let $X$ be a stably locally compact space, $F$ a normalized continuous lower prevision on $X$, and $f$ a bounded continuous function from $X$ to $\mathbb{R}^{+}$. Then there is a normalized continuous linear prevision $G$ such that $F \leq G$ and $F(f)=G(f)$. 
Proof. Recall the function $\breve{F}_{f}$ from the proof of Theorem 3, and define:

$$
\breve{F}_{f}(g)=\inf _{\epsilon \in \mathbb{R}^{+}} \breve{F}_{f+\epsilon}(g)
$$

This is well defined, and is always a non-negative real, never $+\infty$. Indeed, by Claim $\mathrm{H}$ and Claim E, whenever $\epsilon>0, \widetilde{F}_{f+\epsilon}(g) \leq \sup _{x \in X} g(x) \cdot \frac{1}{\epsilon+\inf _{x \in X} f(x)} F(f+\epsilon)<+\infty$.

Claim R. $\breve{F}_{f}(0)=0 . \breve{F}_{f}$ is monotonic. $\breve{F}_{f}$ is positively homogeneous. For every $g \in\left\langle X \rightarrow \mathbb{R}^{+}\right\rangle, \breve{F}_{f}(g) \geq F(g)$.

Proof. By Claim B, Claim C, Claim E, Claim F.

Claim S. Let $F$ be a lower prevision on $X$. If $F$ is normalized, then $\breve{F}_{f+\epsilon}$ is antitone in $\epsilon$.

Proof. Assume $\epsilon<\epsilon^{\prime}$. If $\breve{F}_{f+\epsilon}(g)=+\infty$ (which entails $\epsilon=0$ ), clearly $\breve{F}_{f+\epsilon^{\prime}}(g) \leq$ $\breve{F}_{f+\epsilon}(g)$. So let us assume that $\widetilde{F}_{f+\epsilon}(g)<+\infty$. Recall that:

$$
\breve{F}_{f+\epsilon}(g)=\inf _{\lambda / \lambda(f+\epsilon) \geq g}\left[F(\lambda(f+\epsilon))-\sup _{h \leq \lambda(f+\epsilon)-g} F(h)\right]
$$

Now $F\left(\lambda\left(f+\epsilon^{\prime}\right)\right)=\lambda\left(\epsilon^{\prime}-\epsilon\right)+F(\lambda(f+\epsilon))$ since $F$ is normalized. Moreover, if $g \leq \lambda(f+\epsilon)$, then:

$$
\begin{aligned}
\lambda\left(\epsilon^{\prime}-\epsilon\right)+\sup _{h \leq \lambda(f+\epsilon)-g} F(h) & =\sup _{h \leq \lambda(f+\epsilon)-g} F\left(h+\lambda\left(\epsilon^{\prime}-\epsilon\right)\right) \\
& \quad \text { since } F \text { is normalized } \\
\leq & \sup _{h^{\prime} \leq \lambda\left(f+\epsilon^{\prime}\right)-g} F\left(h^{\prime}\right)
\end{aligned}
$$

since for every $h \leq \lambda(f+\epsilon)-g, h^{\prime}=h+\lambda\left(\epsilon^{\prime}-\epsilon\right)$ is less than or equal to $\lambda\left(f+\epsilon^{\prime}\right)-g$. Therefore:

$$
\begin{aligned}
\breve{F}_{f+\epsilon}(g) & =\inf _{\lambda / \lambda(f+\epsilon) \geq g}\left[F\left(\lambda\left(f+\epsilon^{\prime}\right)\right)-\left[\lambda\left(\epsilon^{\prime}-\epsilon\right)+\sup _{h \leq \lambda(f+\epsilon)-g} F(h)\right]\right] \\
& \geq \inf _{\lambda / \lambda(f+\epsilon) \geq g}\left[F\left(\lambda\left(f+\epsilon^{\prime}\right)\right)-\sup _{h^{\prime} \leq \lambda\left(f+\epsilon^{\prime}\right)-g} F\left(h^{\prime}\right)\right] \\
& \geq \inf _{\lambda / \lambda\left(f+\epsilon^{\prime}\right) \geq g}\left[F\left(\lambda\left(f+\epsilon^{\prime}\right)\right)-\sup _{h^{\prime} \leq \lambda\left(f+\epsilon^{\prime}\right)-g} F\left(h^{\prime}\right)\right] \\
& \quad \breve{F}_{f+\epsilon^{\prime}}(g)
\end{aligned}
$$


Claim T. Let $F$ be a lower prevision on $X$. If $F$ is normalized, then $\breve{F}_{f}$ is convex. Proof. For every $\epsilon^{\prime}, \epsilon^{\prime \prime} \geq 0$, there is $\epsilon \geq 0$ such that $\widetilde{F}_{f+\epsilon}(g)+\breve{F}_{f+\epsilon}\left(g^{\prime}\right) \leq$ $\breve{F}_{f+\epsilon^{\prime}}(g)+\breve{F}_{f+\epsilon^{\prime \prime}}\left(g^{\prime}\right)$. Indeed, by Claim S, it is enough to take $\epsilon=\max \left(\epsilon^{\prime}, \epsilon^{\prime \prime}\right)$. So $\inf _{\epsilon \geq 0}\left[\widetilde{F}_{f+\epsilon}(g)+\widetilde{F}_{f+\epsilon}\left(g^{\prime}\right)\right] \leq \inf _{\epsilon^{\prime} \geq 0} \breve{F}_{f+\epsilon^{\prime}}(g)+\inf _{\epsilon^{\prime \prime} \geq 0} \breve{F}_{f+\epsilon^{\prime \prime}}\left(g^{\prime}\right)$. It follows:

$$
\begin{aligned}
\breve{F}_{f}\left(g+g^{\prime}\right) & =\inf _{\epsilon \geq 0} \breve{F}_{f+\epsilon}\left(g+g^{\prime}\right) \\
& \leq \inf _{\epsilon \geq 0}\left[\breve{F}_{f+\epsilon}(g)+\breve{F}_{f+\epsilon}\left(g^{\prime}\right)\right] \quad \text { by Claim D and Claim E } \\
& \leq \inf _{\epsilon^{\prime} \geq 0} \breve{F}_{f+\epsilon^{\prime}}(g)+\inf _{\epsilon^{\prime \prime} \geq 0} \breve{F}_{f+\epsilon^{\prime \prime}}\left(g^{\prime}\right) \\
& =\breve{F}_{f}(g)+\breve{F}_{f}\left(g^{\prime}\right)
\end{aligned}
$$

$\breve{F}_{f}$ is therefore sub-linear, hence convex since it is positively homogeneous by Claim R.

Claim U. Let $F$ be a lower prevision on $X$. If $F$ is normalized, then $\widetilde{F}_{f}(f)=F(f)$. Proof. Clearly $\breve{F}_{f}(g) \leq \breve{F}_{f}(g)$ (take $\epsilon=0$ ). When $g=f$, by Claim G $\breve{F}_{f}(f)=$ $F(f)$. So $\breve{F}_{f}(f) \leq F(f)$. The converse inequality follows by Claim U.

Claim V. Let $F$ be a lower prevision on $X$. If $F$ is normalized, then $\breve{F}_{f}\left(\chi_{X}\right)=1$. Proof. If there is no $\lambda$ such that $1 \leq \lambda(f+\epsilon)$,

$$
\begin{aligned}
\breve{F}_{f+\epsilon}\left(\chi_{X}\right) & =\inf _{\lambda / 1 \leq \lambda(f+\epsilon)}\left[F(\lambda(f+\epsilon))-\sup _{h \leq \lambda(f+\epsilon)-1} F(h)\right] \\
& =\inf _{\lambda / 1 \leq \lambda(f+\epsilon)}\left[F(\lambda(f+\epsilon)-1)+1-\sup _{h \leq \lambda(f+\epsilon)-1} F(h)\right]
\end{aligned}
$$

since $F$ is normalized. Since $\sup _{h \leq \lambda(f+\epsilon)-1} F(h)=F(\lambda(f+\epsilon)-1), \breve{F}_{f+\epsilon}\left(\chi_{X}\right)=$ 1. On the other hand, if there is no $\lambda$ such that $1 \leq \lambda(f+\epsilon)$, then $\breve{F}_{f+\epsilon}\left(\chi_{X}\right)=+\infty$. This can only happen when $\epsilon=0$, and provided $\inf _{x \in X} f(x)=0$. In this case, $\breve{F}\left(\chi_{X}\right)$ is the greatest lower bound of 1 and $+\infty$; otherwise, $\breve{F}\left(\chi_{X}\right)$ already equals 1 , directly.

Let us prove the Theorem. Since $F$ is super-linear, $\breve{F}_{f}$ is sub-linear by Claim T, $F$ is monotonic and $\breve{F}_{f}$ also by Claim R, we can apply Roth's Sandwich Theorem: there is a functional $G_{0}$ from $\left\langle X \rightarrow \mathbb{R}^{+}\right\rangle$to $\overline{\mathbb{R}}^{+}$, which is monotonic and linear, and such that $F \leq G_{0} \leq \breve{F}_{f}$. By Claim U, $F(f) \leq G_{0}(f) \leq \breve{F}_{f}(f)=F(f)$, so $G_{0}(f)=$ $F(f)$. Next, $\breve{F}_{f}$ takes its values in $\mathbb{R}^{+}$, so $G_{0}$ too. In particular, $G_{0}$ is a prevision. $G_{0}$ is normalized, since for every bounded continuous function $g$, for every $a \in \mathbb{R}^{+}$, 
$G_{0}(a+g)=a G_{0}\left(\chi_{X}\right)+G_{0}(g)$ (since $G_{0}$ is linear) $=a+G_{0}(g)$. Indeed, $1=F\left(\chi_{X}\right) \leq$ $G_{0}\left(\chi_{X}\right) \leq \breve{F}_{f}\left(\chi_{X}\right)=1$ by Claim V.

So $G_{0}$ is a normalized linear prevision such that $F \leq G_{0}$ et $F(f)=G_{0}(f)$. (And this holds on any topological space $X$.) Now $X$ is stably locally compact, hence stably core compact, so let $G=\mathfrak{r}\left(G_{0}\right)$. By Claim $\mathrm{Q}, G$ is a continuous linear prevision. Since $G$ is the greatest continuous functional below $G_{0}$ and $F \leq G_{0}$, it follows that $F \leq G$. Since $G \leq G_{0}, G(f) \leq G_{0}(f)=F(f) \leq G(f)$, so $G(f)=F(f)$. Finally, we show that $G$ is normalized-and we do not need $X$ to be compact, as Claim Q would suggest. We claim indeed that $G(a+g)=a+G(g)$ : by linearity, $G(a+g)=$ $a G\left(\chi_{X}\right)+G(g)$, and $G\left(\chi_{X}\right)=1$ since $G\left(\chi_{X}\right) \leq G_{0}\left(\chi_{X}\right)=1$ on the one hand, and $G\left(\chi_{X}\right) \geq F\left(\chi_{X}\right)=1$ on the other hand.

Theorem 5. Let $X$ be stably locally compact, $F$ a continuous normalized prevision on $X$. Then $F$ is lower iff CCoeur $1(F) \neq \emptyset$ and for every $f \in\left\langle X \rightarrow \mathbb{R}^{+}\right\rangle, F(f)=$ $\inf _{G \in C C_{\text {Courr }}(F)} G(f)$. In this case, the inf is attained: $F(f)=\min _{G \in C C_{\text {Cour }}(F)} G(f)$.

Proof. If $F$ is lower then $C C_{\text {Coeur }}(F)$ is non-empty by Theorem 4. Moreover, for every $f \in\left\langle X \rightarrow \mathbb{R}^{+}\right\rangle$, there is a $G \in C$ Coeur $_{1}(X)$ such that $F(f)=G(f)$, so clearly $F(f)=\inf _{G \in C_{\text {Coeur }}(F)} G(f)$ and the inf is attained.

Conversely, if $C$ Coeur $1(F)$ is non-empty and $F(f)=\inf _{G \in C C_{\text {Coeur }}(F)} G(f)$ for every $f \in\left\langle X \rightarrow \mathbb{R}^{+}\right\rangle$, then $F$ is super-additive, hence lower. Indeed:

$$
\begin{aligned}
& F\left(f+f^{\prime}\right)=\inf _{G \in \text { Coeur }_{1}(F)} G\left(f+f^{\prime}\right)=\inf _{G \in \text { Coeur }_{1}(F)}\left(G(f)+G\left(f^{\prime}\right)\right) \\
& \geq \inf _{G \in \text { Coeur }_{1}(F)} G \text { is additive } \\
& \inf _{G \in \text { Coeur }_{1}(F)} G\left(f^{\prime}\right)=F(f)+F\left(f^{\prime}\right)
\end{aligned}
$$

Proposition 4. Let $X$ be stably compact, $F$ a normalized continuous lower prevision, then CCoeur $r_{1}(F)$ is a non-empty saturated compact convex subset of $\mathbf{P}_{1 w k}^{\triangle}(X)$.

Proof. Convexity is obvious, non-emptiness is by Theorem 5, and saturation is clear. Compactness is almost a consequence of [19, Corollary 2]. We would need to check that $C=\left\langle X \rightarrow \mathbb{R}^{+}\right\rangle$is a so-called continuous d-cone, with an additive way-below relation. (Note that what Plotkin calls weak* ${ }^{*}$-Scott what we simply call the weak topology.) Claim $\mathrm{M}$ indeed shows that $\ll$ is additive, however every (continuous) d-cone is a (continuous) cpo, which $\left\langle X \rightarrow \mathbb{R}^{+}\right\rangle$fails to be. (One might argue we could have defined previsions as taking maps from $\left\langle X \rightarrow \overline{\mathbb{R}}^{+}\right\rangle$to $\overline{\mathbb{R}}^{+}$instead. However, we wanted to provide a nice link with the theory of games and belief functions, and the latter works more smoothly provided we exclude $+\infty$. Moreover, in applications we only really need sub-normalized or normalized games/previsions.)

However, here is a direct argument, inspired by [9]. We need to recall that the cocompact topology of a stably compact space $X$ is the collection of all complements of saturated compacts of $X$. The space $X$ with its co-compact topology is its de Groot dual $X^{d}$. Then $X^{d}$ is also stably compact, its specialization ordering is the converse 
$\geq$ of that of $X, X^{d d}=X$. The coarsest topology containing both the original and the co-compact topology is the patch topology. $X$ equipped with the patch topology is a compact pospace, which we write $X^{\prime}$ [9]. Note that the product $Y=\prod_{i \in I} Y_{i}$ of stably compact spaces is stably compact, that $Y^{\prime}=\prod_{i \in I} Y_{i}^{\prime}$, and that the specialization ordering of $Y$ is the component-wise ordering.

The space $[0,1]$ is stably compact, and $[0,1]^{\prime}$ is equipped with the usual, metric topology. Let $X$ be stably compact, and consider $Z=\prod_{\substack{f \in\left\langle X \rightarrow \mathbb{R}^{+}\right\rangle \\ \sup _{x \in X} f(x)=1}}[0,1]$, which is

also stably compact. Let $P(X)$ be any of the spaces of sub-normalized previsions we consider. There is an obvious map $e: P(X) \rightarrow Z$ that sends $F$ to the family of all $F(f)$, $f \in\left\langle X \rightarrow \mathbb{R}^{+}\right\rangle, \sup _{x \in X} f(x)=1$. Conversely, for any family $z=\left(z_{f}\right)_{f \in\left\langle X \rightarrow \mathbb{R}^{+}\right\rangle}$

of elements of $[0,1]$, one may define a positively homogeneous functional $m(z)$ from $\left\langle X \rightarrow \mathbb{R}^{+}\right\rangle$to $\mathbb{R}^{+}$by: $m(z)(0)=0, m(z)(f)=a z_{f / a}$ when $a=\sup _{x \in X} f(x)>0$.

We then check that the subspace of $Z$ of those $z$ such that $m(z)$ is a (plain, lower, upper, sub-normalized, normalized) prevision is patch-closed in $Z$, hence stably compact. (See [7, lemme 11.6.1].) The idea is that this subspace is defined as the set of objects $z$ satisfying a collection of patch-continuous equations, i.e., equations of the form $f\left(z_{f_{1}}, \ldots, z_{f_{n}}\right)=g\left(z_{f_{1}}, \ldots, z_{f_{n}}\right)$, where $f_{1}, \ldots, f_{n}$ are fixed, and $f$ and $g$ are continuous functions from $[0,1]^{\prime n}$ to $[0,1]^{\prime}$. E.g., that $m(z)$ is monotonic is equivalent to the fact that $a \times z_{f / a} \leq b \times z_{g / b}$ for all $f, g \in\left\langle X \rightarrow \mathbb{R}^{+}\right\rangle$that are not identically zero and such that $f \leq g$, where $a=\sup _{x \in X} f(x), b=\sup _{x \in X} g(x)$; in turn, an inequality $u \leq v$ is an abbreviation for the equation $\max (u, v)=v$. Since every intersection of patch-closed subsets is patch-closed, the given subspace is patch-closed.

We then check that, if $Z_{\leq 1}$ is the subspace of $Z$ consisting of all $z$ such that $m(z)$ is a sub-normalized prevision, then $e$ defines a homeomorphism of the space $P_{\leq 1} w k(X)$ of all sub-normalized previsions onto $Z_{\leq 1}$, with inverse $m$. In particular, $P_{\leq 1 w k}(X)$ is stably compact.

The technique of patch-continuous equations implies that given any conjunction of properties among "lower", "upper", "linear" and "normalized", the subspace of those previsions in $P_{\leq 1 w k}(X)$ satisfying these properties is also patch-closed in it, hence stably compact.

Next, we observe that $\mathfrak{r}: P_{\leq 1 w k}(X) \rightarrow \mathbf{P}_{\leq 1 w k}(X)$ (see Claim Q and later) and the obvious inclusion $\mathfrak{s}: \mathbf{P}_{\leq 1} w k(X) \rightarrow P_{\leq 1} w k(X)$ forms a retraction-section pair. I.e., that they are continuous and $\mathfrak{r}(\mathfrak{s}(F))=F$ for all $F$. This allows us to conclude that $\mathbf{P}_{\leq 1 w k}(X)$ is stably compact: by Lawson's Lemma (see again [9]), any retract of a stably compact space is stably compact. We need $X$ to be compact additionally, in the case of normalized previsions, to be able to apply Claim $\mathrm{Q}$ and show that $\mathfrak{r}$ : $P_{1 w k}(X) \rightarrow \mathbf{P}_{1 w k}(X)$.

Finally, we note that $C$ Coeur $1(X)$ is the intersection of $\uparrow F$ (the set of all normalized continuous previsions $F^{\prime}$ such that $\left.F \leq F^{\prime}\right)$ and $\mathbf{P}_{1 w k}^{\triangle}(X)$. The former is trivially compact (every set $\uparrow x$ is compact in any topological space), hence patch-closed in $\mathbf{P}_{1 w k}(X)$. The technique of patch-continuous equations shows that $\mathbf{P}_{1 w k}^{\Delta}(X)$ is again patch-closed in $\mathbf{P}_{1 w k}(X)$, so CCoeur $1(X)$ is patch-closed in $\mathbf{P}_{1 w k}^{\triangle}(X)$. It is easy to see that a patch-closed subset of a stably compact space is compact. 
Proposition 5. Let $X$ be a topological space, $F$ a normalized continuous upper prevision, then $C \operatorname{Peau}_{1}(F)$ is a closed convex subset of $\mathbf{P}_{1 \text { wk }}^{\triangle}(X)$. It is non-empty as soon as $X$ is stably compact.

Proof. Convexity is again obvious. We have already mentioned that $C P e a u_{1}(F)$ would be non-empty if $X$ is stably compact (details in [7, théorème 11.7.4]). Then we notice that:

$$
\begin{aligned}
\operatorname{CPeau}_{1}(X) & =\left\{G \in \mathbf{P}_{1 w k}^{\triangle}(X) \mid G \leq F\right\}=\bigcap_{f \in\left\langle X \rightarrow \mathbb{R}^{+}\right\rangle}\left\{G \in \mathbf{P}_{1 w k}^{\triangle}(X) \mid G(f) \leq F(f)\right\} \\
& =\bigcap_{f \in\left\langle X \rightarrow \mathbb{R}^{+}\right\rangle}\left(\mathbf{P}_{1 w k}^{\triangle}(X) \backslash[f>F(f)]\right)
\end{aligned}
$$

which is therefore closed in the weak topology.

Proposition 6. Let $X$ be a stably compact space. The continuous normalized body $C_{C o r p s}(F)=C C_{1}$ oeur ${ }_{1}\left(F^{-}\right) \cap C_{P e a u}\left(F^{+}\right)$of a continuous normalized fork $F=\left(F^{-}, F^{+}\right)$on $X$ is a lens. Moreover, CCoeur ${ }_{1}\left(F^{-}\right)=\uparrow C_{C o r p s}(F)$ and $C \operatorname{Peau}_{1}\left(F^{+}\right)=\downarrow \operatorname{CCorps}_{1}(F)$.

Proof. We show that: $(*)$ whenever $G \in C$ Coeur $_{1}\left(F^{-}\right)$, there is some $G^{\prime} \in C C_{\text {oeur }}\left(F^{-}\right) \cap$ $C P e a u_{1}\left(F^{+}\right)$such that $G^{\prime} \leq G$.

Let $F^{\prime}(h)=\inf _{\substack{f, g \in\left\langle X \rightarrow \mathbb{R}^{+}\right\rangle \\ f+g \geq h}}\left(F^{+}(f)+G(g)\right)$. This is well-defined since e.g., we may take $f=0$ and $g=h$. This also implies that $F^{\prime}(h) \leq G(h)$. Clearly, $F^{-} \leq F^{\prime} \leq$ $G$ : we have just shown $F^{\prime} \leq G$, and for the other inequality, we note that for every $f$, $g$ with $f+g \geq h, F^{+}(f)+G(g) \geq F^{+}(f)+F^{-}(g)$ (by assumption) $\geq F^{-}(f+g)$ (by (3)) $\geq F^{-}(h)$.

Now we observe that $F^{\prime}$ is an upper prevision. Indeed, $F^{\prime}(0)=0$ (taking $f=g=$ 0 ), and when $\alpha>0, F^{\prime}(\alpha h)=\inf _{f+g \geq \alpha h}\left(F^{+}(f)+G(g)\right)=\inf _{f^{\prime}+g^{\prime} \geq h}\left(F^{+}\left(\alpha f^{\prime}\right)+\right.$ $G(\alpha g))=\alpha F^{\prime}(h)$; so $F^{\prime}$ is positively homogeneous. $F^{\prime}$ is clearly monotonic, while:

$$
\begin{aligned}
F^{\prime}(h)+F^{\prime}\left(h^{\prime}\right) & =\inf _{f+g \geq h, f^{\prime}+g^{\prime} \geq h^{\prime}}\left(F^{+}(f)+G(g)+F^{+}\left(f^{\prime}\right)+G\left(g^{\prime}\right)\right) \\
& \geq \inf _{f+g \geq h, f^{\prime}+g^{\prime} \geq h^{\prime}}\left(F^{+}\left(f+f^{\prime}\right)+G\left(g+g^{\prime}\right)\right) \\
& \geq \inf _{f^{\prime \prime}+g^{\prime \prime} \geq h+h^{\prime}}\left(F^{+}\left(f^{\prime \prime}\right)+G\left(g^{\prime \prime}\right)\right)=F^{\prime}\left(h+h^{\prime}\right)
\end{aligned}
$$

So $F^{\prime}$ is upper.

Using Roth's Sandwich Theorem again, there is a linear monotonic functional $G_{0}$ such that $F^{-} \leq G_{0} \leq F^{\prime}$. Because $G_{0} \leq F^{\prime}, G_{0}$ does not take the value $+\infty$, so $G_{0}$ is a linear prevision. Let $G^{\prime}=\mathfrak{r}\left(G_{0}\right)$. By Claim $\mathrm{Q}, G$ is a continuous linear prevision, and $F^{-} \leq G^{\prime} \leq F^{\prime}$, as above. In particular, $1=F^{-}\left(\chi_{X}\right) \leq G^{\prime}\left(\chi_{X}\right) \leq F^{\prime}\left(\chi_{X}\right)=1$, so $G^{\prime}\left(\chi_{X}\right)=1$; using the fact that $G^{\prime}$ is linear, $G^{\prime}$ is then normalized. Since $F^{-} \leq G^{\prime}$, $G^{\prime} \in C_{C o e u r}\left(F^{-}\right)$. Since $G^{\prime} \leq F^{\prime}$ and clearly $F^{\prime} \leq F^{+}, G^{\prime} \in \operatorname{CPeau}_{1}\left(F^{+}\right)$. Finally, since $G^{\prime} \leq F^{\prime}$ and $F^{\prime} \leq G, G^{\prime} \leq G$. So $(*)$ obtains. 
It follows in particular that $\operatorname{CCoeur}_{1}\left(F^{-}\right) \cap C P e a u_{1}\left(F^{+}\right)$is non-empty. That $\operatorname{CCoeur}_{1}\left(F^{-}\right)=\uparrow\left(\operatorname{CCoeur}_{1}\left(F^{-}\right) \cap C \operatorname{Peau}_{1}\left(F^{+}\right)\right)$is an easy consequence of $(*)$. That $C \operatorname{Peau}_{1}\left(F^{+}\right)=\downarrow\left(\right.$ CCoeur $\left._{1}\left(F^{-}\right) \cap C \operatorname{Peau}_{1}\left(F^{+}\right)\right)$can be shown in a similar way, by defining $F^{\prime \prime}(h)=\sup _{f, g \in\left\langle X \rightarrow \mathbb{R}^{+}\right\rangle}\left(F^{-}(f)+G(g)\right)$, where $G \in C \operatorname{Peau}_{1}\left(F^{-}\right)$, and using $F^{\prime \prime}$ to show that there is some $G^{\prime} \in \operatorname{CCoeur}_{1}\left(F^{-}\right) \cap \operatorname{CPeau}_{1}\left(F^{+}\right)$such that $G \leq G^{\prime}$. 